(Aus dem physiologischen Institut der Universität Königsberg i. Pr.)

\title{
Untersuchungen über indirekte Muskelreizung durch abgebrochene Kondensatorentladungen.
}

Von

\section{Hermann.}

Nach Versuchen von Alfred Adam.

(Mit 2 Textfiguren.)

\section{Einleitung and Versuchsverfahren.}

In meiner letzten Arbeit über indirekte Muskelreizung durch Kondensatorentladungen ${ }^{x}$ ) habe ich auf Grund meiner Versuche die Ansicht ausgesprochen, dass nur ein Bruchteil der elektrischen Energie zur Wirkung gelangt, nämlich derjenige, welcher in eine bestimmte "kritische" Zeit fällt, von der Grössenordnung der Latenzzeit des Muskelelements, nämlich etwa 0,0012-0,0024 sek. ${ }^{2}$ ). Zur Prüfung dieser Ansicht hat L. L a pi cqu e ${ }^{3}$ ) den sinnreichen Gedanken ausgeführt, die den Minimaleffekt gebende Entladung sich nicht vollständig vollziehen zu lassen, sondern abzubrechen, und zwar soweit wie es ohne Ausbleiben des Effektes möglich ist, wozu er sich des Schussrheotoms von G. We iss bediente. Die Zeit, auf welche Lapicque die Entladung einschränken konnte, nennt er „temps utile“, wofür ich hier "Nutzzeit" sagen werde; diese Zeit findet er nicht konstant, wie ich es für meine kritische Zeit angenommen hatte, sondern um so kürzer je kleiner die Kapazität,

1) Dieses Archiv Bd. 111 S. 537. 1906.

2) Eine ähnliche Zeitgrösse hatten schon Cybulski \& Zanietowski als die dem Optimum der Kapazität entsprechende Entladungszeit gefunden, und G. Weis s hatte bei Zeitschliessungen bemerkt, dass nach einer ähnlichen Zeit der Strom zu wirken aufhört (s. a. a. O. S. 552, 566).

3) Compt. rend. de la soc. d. biol. 1907 t. 1 p. 701. 
für höhere Kapazitäten im übrigen von derselben Grössenordnung, wie ich sie angenommen hatte. Ich will gleich hier bemerken, dass dies Ergebnis sich mit meiner Aufstellung sehr gut vereinigen lässt, wenn man berücksichtigt, dass kleinere Kapazitäten höheres Potential, also stärkeren Anfangsreiz erfordern, stärkere Reize aber nach einer Reihe von Untersuchungen die Tendenz haben, die Latenzzeit zu verkürzen.

Zum besseren Verständnis reproduziere ich hier die Versuchsreihe von Lapicque (leider die einzige von ihm mitgeteilte).

Widerstand $700000 \mathrm{hm}=7 \cdot 10^{13} \mathrm{~cm} / \mathrm{sek}$.

\begin{tabular}{|c|c|c|}
\hline $\begin{array}{c}\text { Kapazität } \\
c\end{array}$ & $\begin{array}{c}\text { Potential } \\
p\end{array}$ & $\begin{array}{c}\text { Nutzzeit } \\
t\end{array}$ \\
\hline $\begin{array}{ll}1 & \text { Mf. } \\
0,1 & " \\
0,05 & " \\
0,02 & " \\
0,01 \quad "\end{array}$ & $\begin{array}{cc}0,106 & \text { Volt } \\
0,123 & " \\
0,140 & " \\
0,185 & " \\
0,255 & "\end{array}$ & $\begin{array}{l}\left.1,81 \sigma^{1}\right) \\
1,54 " \\
1,35 " \\
0,93 " \\
0,63 "\end{array}$ \\
\hline
\end{tabular}

$p$ ist das bei vollständiger Entladung zur Minimalzuckung erforderliche Potential, welches dann in den Versuchen mit abgebrochener Entladung beibehalten wird.

Man kann nun für jeden Einzelversuch berechnen:

1. bis zu welcher Höhe $p^{*}$ das Anfangspotential $p$ durch die Entladung gesunken ist,

2. die anfangs vorhandene Elektrizitätsmenge $Q$ und den durch die Entladung ausgegebenen Teil $Q^{*}$ derselben,

3. die anfangs vorhandene Energie $E$ und den durch die Entladung ausgegebenen Teil $E^{*}$ derselben.

Es ist nämlich, wenn der Entladungskreis von Selbstinduktion und Kapazität frei ist oder diese vernachlässigt werden dürfen:

$$
\left.\begin{array}{c}
p^{*}=p e^{-\frac{t}{w c}}, \quad Q=p c, \quad Q^{*}=p c\left(1-e^{-\frac{t}{w c}}\right), \\
E=\frac{1}{2} p^{2} c, \quad E^{*}=\frac{1}{2} p^{2} c\left(1-e^{-\frac{2 t}{w c}}\right) .
\end{array}\right\} \ldots
$$

L a p i c que hat nun zwar für seine Versuchsbedingungen (auf weiter unten anzugebendem Wege) gefunden, dass diese Vernachlässigung nicht erlaubt ist, so dass die nach den vorstehenden Formeln be-

1) $\sigma$ bedeutet in dieser Arbeit durchweg 0,001 Sekunde. 
rechneten Zahlen nur Näherungswerte darstellen; trotzdem sind dieselben von Interesse. Sie lauten

$\left(1\right.$ Volt-Mf. $=1$ Mikrocoulomb, 1 Volt ${ }^{2}$-Mf. $=10$ Erg $):$

\begin{tabular}{|c|c|c|c|c|c|c|c|c|c|}
\hline \multirow[b]{2}{*}{$\begin{array}{c}\text { Kapazität } \\
c \\
\text { Mikrofar. }\end{array}$} & \multirow[b]{2}{*}{$\begin{array}{c}\text { Nutz- } \\
\text { zeit } t \\
\sigma\end{array}$} & \multicolumn{2}{|c|}{ Potential } & \multicolumn{3}{|c|}{ Elektrizitätsmenge } & \multicolumn{3}{|c|}{ Energie } \\
\hline & & $\begin{array}{c}\text { an- } \\
\text { fangs } \\
p_{\mathrm{V}}\end{array}$ & \begin{tabular}{|c|}
$\begin{array}{c}\text { zu- } \\
\text { letzt } \\
p^{*}\end{array}$ \\
olt
\end{tabular} & $\begin{array}{c}\text { vor- } \\
\text { handen } \\
Q \\
\text { Hiund } \\
\text { Mikro }\end{array}$ & $\begin{array}{c}\text { abge- } \\
\text { geben } \\
Q^{*} \\
\text { oulstel } \\
\text { oulomb }\end{array}$ & $\frac{Q^{*}}{Q}$ & $\begin{array}{c}\text { vor- } \\
\text { handen } \\
E \\
\text { Hundert }\end{array}$ & $\begin{array}{c}\text { abge- } \\
\text { geben } \\
\boldsymbol{H}^{*} \\
\\
\text { tel Erg }\end{array}$ & $\frac{E^{*}}{E}$ \\
\hline $\begin{array}{l}1,0 \\
0,1 \\
0,05 \\
0,02 \\
0,01\end{array}$ & $\begin{array}{l}1,81 \\
1,54 \\
1,35 \\
0,93 \\
0,63\end{array}$ & $\begin{array}{l}0,106 \\
0,123 \\
0,140 \\
0,185 \\
0,255\end{array}$ & $\begin{array}{l}0,1033 \\
0,0987 \\
0,0952 \\
0,0951 \\
0,1037\end{array}$ & $\begin{array}{r}10,60 \\
1,23 \\
0,70 \\
0,37 \\
0,26\end{array}$ & $\begin{array}{l}0,271 \\
0,243 \\
0,224 \\
0,180 \\
0,151\end{array}$ & $\begin{array}{l}0,0255 \\
0,1975 \\
0,3199 \\
0,4854 \\
0,5934\end{array}$ & $\begin{array}{l}5,618 \\
0,756 \\
0,490 \\
0,342 \\
0,325\end{array}$ & $\begin{array}{l}0,146 \\
0,134 \\
0,132 \\
0,126 \\
0,135\end{array}$ & $\begin{array}{l}0,0504 \\
0,3560 \\
0,5375 \\
0,7352 \\
0,8347\end{array}$ \\
\hline
\end{tabular}

Eine Betrachtung dieser Zahlen ergibt folgendes:

1. Die Werte $p, Q$ und $E$ verhalten sich so, wie es früheren Untersuchungen entspricht; dass sich bei $E$ kein Minimum zeigt, hat sicher nur darin seinen Grund, dass der Versuch bei 0,01 Mf. abbricht und das Minimum meist zwischen 0,01 und 0,001 Mf. liegt.

2. Die Werte $p^{*}$ sind auffallend konstant; sie variieren nur òis $\pm 4,5 \%$ ihres Mittelwertes. Dies kann aber unmöglich so gedeutet werden, dass etwa zum Effekt bei jeder Kapazität die Entladung so lange dauern muss, bis ein bestimmter Potentialwert erreicht ist; wenigstens wäre ein $\mathrm{zu}$ dieser Konsequenz fübrendes Erregungsgesetz schwer auszudenken.

3. Von der im Kondensator aufgespeicherten Elektrizitätsmenge $Q$. wird ein um so kleinerer Teil verausgabt, je grösser die Kapazität; bei der grössten beträgt dieser Teil nur 2,55, bei der kleinsten $59 \%$. Der nicht verbrauchte Teil würde den Effeǩt nicht im mindesten begünstigt baben, denn die Ladungen sind grade für den Minimaleffekt ausprobiert und doch wird der Effekt selbst durch beträchtliche Verkürzungen der Entladung nicht verhindert, solange diese nicht die im Versuch festgestellte Grenze überschreitet. Man hätte erwarten können, dass schon die geringste Verkürzung der Entladung jeden Effekt verhindert hätte.

4. Die zur Erregung wirklich verbrauchte Quantität $Q^{*}$ ist um so geringer, je kleiner die Kapazität.

5. Auffallend ist ferner die Konstanz ner Werte $\boldsymbol{E}^{*}$, deren Variation nur bis $\pm 5,7 \%$ ihres Mittels geht, obwohl sie ein Mini- 
mum durchlaufen. Man köunte also, vorbehaltlich weiterer Prüfung, folgern, dass bei jeder Kapazität die Entladung so lange fortgesetzt werden muss, bis ein ganz bestimmtes Quantum Energie hergegeben ist. Übrigens wird auch von der aufgespeicherten Energie nur ein Bruchteil zur Erregung verwendet, der von 5\% (bei $1 \mathrm{Mf}$.) bis $83 \%$ (bei $0,01 \mathrm{Mf}$.) geht.

Bemerkenswert ist, dass der Versuch von Lapi eque die Ansicht Hoorweg's, dass die Kondensatorversuche sein Erregungsgesetz bestätigen, auf das Bündigste widerlegt. Denn Hoorweg berechnet das zur Erregung erforderliche Ladungspotential aus seiner Theorie, dass die differentielle Erregung dem Betrage $i e^{-\beta t}$ proportional sei, rlurch eine Integration bis $t=\infty$, und findet das Ergebnis mit den Versuchen in glänzender Übereinstimmung, während Lapicque unwiderleglich gezeigt hat, dass nur der bis zu einem sehr kleinen Werte von $t$ entladene Anteil in Rechnung gezogen werden darf. Die scheinbare Übereinstimmung war also trügerisch.

Verfahren zu weiteren Versuchen über den Quantitätsund Energiebedarf für Minimalzuckungen.

Fortan haben offenbar alle Versuche mit vollständigen Kondensatorentladungen für die Frage des Erregungsgesetzes nur noch geringes Interesse, da wir nun weit bestimmter wissen, als ich es in meiner letzten Arbeit begründen konnte, dass hier weit mehr Quantität und Energie aufgewendet wird, als zur Wirkung kommt. Dagegen schien es wichtig, den Versuch von Lapicque zu wiederholen, zumal, wie soeben gezeigt worden ist, derselbe die Aussicht eröffnet, den wirklichen Bedarf an Quantität und Energie bei Reizung des Muskels durch Kondensatorentladungen festzustellen. Diese Versuche hat Herr Alfred A d a m unter meiner Mitwirkung ausgeführt.

Statt des Schussverfahrens von Weiss, welches zweifellos selır grosse Anforderungen an die Zeit und Geduld des Experimentators stellt, verwendeten wir jedoch das Helmholtz'sche Pendel in der vortrefflichen Ausführung von Edelmann. Dieser Apparat arbeitet wobl ebenso genau wie das Schussrheotom, ist viel bequemer $z u$ handhaben und erfordert, worauf es hier vor allem ankommt, für den Ablauf einer Versuchsreihe zweifellos viel weniger Zeit.

Wir begannen mit Versuchen der Lapicque'schen Art; sehr bald aber erwies es sich als wenig zweckmässig, bei gegebener E. Pflüger, Archiv für Physiologie. Bd. 127. 
Ladung die erforderliche Entladungszeit auszuprobieren, weil ein möglichst schneller Ablauf des Versuchs dringend wünschenswert ist, da das Präparat seine Erregbarkeit und sogar seinen Widerstand beständig ändert. Hierin liegt, wie jeder Arbeiter auf diesem Gebiete bestätigen wird, die Ursache der grossen Fehlerbreiten solcher Versuche, welche es allein erklärlich macht, dass so viele sorgfältige Experimentatoren die verschiedensten Gesetze aufstellen und mit ibren Ergebnissen vereinbar finden konnten. Eine andere, kaum geringere Quelle der Ungenauigkeit liegt darin; dass das Urteil, ob die Minimalzuckung vorliegt oder nicht, allzu häufig nur mit einiger Willkür gefällt werden kann. Den letzteren Übelstand haben wir auch diesmal, wie sehon in meiner letzten Arbeit, dadurch etwas zu vermindern gesucht, dass die Zuckungen nicht am ganzen Unterschenkel, sondern am blossen Gastroknemius, mittels des Zuckungstelegraphen, beobachtet wurden.

Da man viel rascher an einem Rheostaten durch Stöpseln Widerstände ausprobieren kann, als mittels der Mikrometerschraube des Kontaktschlittens Entladungszeiten, so haben wir die Versuche so umgeändert, dass für gegebene Entladungszeiten bei jeder Kapazität das zur Minimalzuckung nötige Ladungspotential ermittelt wurde. Dies hat ausser dem schnellen Ablauf des Versuchs den grossen Vorteil, dass die zum Ausprobieren von Zeiten erforderlichen beständigen Schlittenverschiebungen, welche für die feine Mikrometerschraube gefährlich sind, vermieden werden.

Das Schema Fig. 1 verdeutlicht die Versuchsanordnung. Die Kette $\boldsymbol{A}$ besteht aus einem Akkumulatorenelement oder (bei len kürzesten Entladungszeiten oder kleinsten Kapazitäten) aus mehreren ${ }^{1}$ ). $O_{1}$ and $O_{2}$ sind die beiden auf Schlitten mikrometrisch verschiebbaren Öffnungskontakte, welche das Pendel nacheinander öffnet. $O_{1}$ ist - in den Kettenkreis, $O_{2}$ in den Kondensatorkreis eingeschaltet; der Schlüssel $T$ gestattet, durch sein Geschlossensein die Öffnung des Kondensatorkreises zu beseitigen, so dass die Entladuug sich vollständig vollzieht. $K$ ist ein Edelmann'scher PräzisionsGlimmerkondensator; sein Kreis enthält ausser dem Nerven $N$ noch ein ballistisches (Drehspulen-)Galvanometer $G$ (s. unten) und meist einen $\mathrm{Zusatz}$ widerstand $W$ von $100000 \mathrm{Ohm}$. Derselbe dient einerseits dazu, die unvermeidlichen Widerstandsänderungen der

1) Die Elemente waren stets voll, also auf 2 Volt geladen. 
Nervenstrecke während der Versuchreihe weniger schädlich $\mathrm{zu}$ machen, andererseits dazu, den Einfluss der Selbstinduktion (s. unten) zu vermindern. Der Schlüssel $S$ wurde jedesmal geschlossen, ehe $O_{1}$ geschlossen wurde und dann sofort wieder geöffnet; er dient nur dazu, dem Präparat die Ladezuckung zu ersparen.

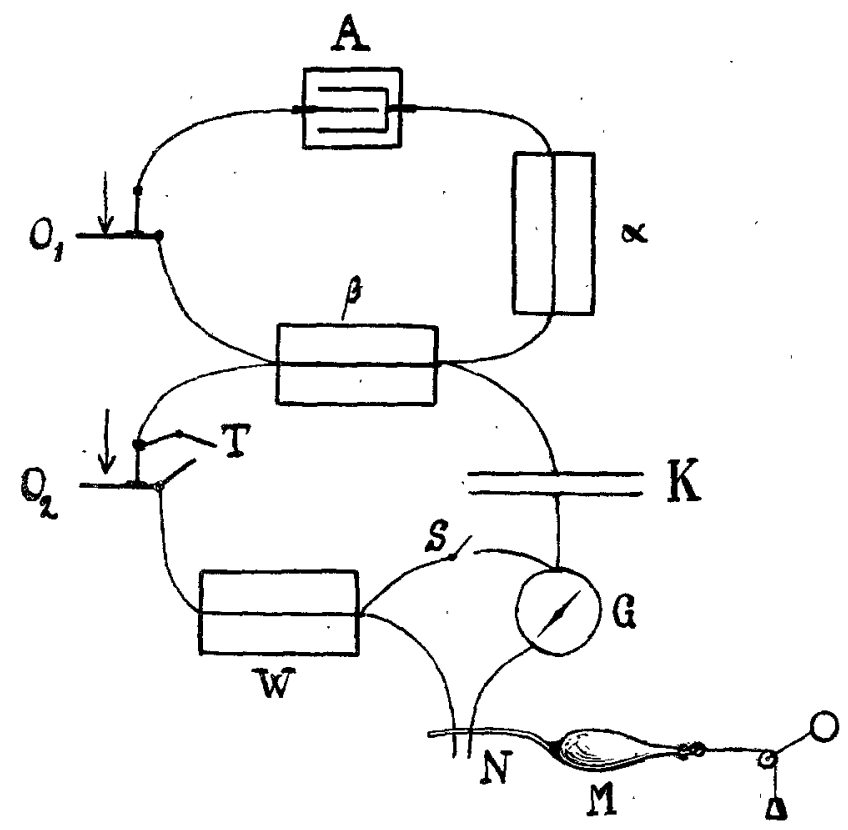

Fig. 1.

Die Eichung des Pendelapparats auf die Nullstellung (d. b. diejenige, bei welcher $O_{2}$ gleichzeitig mit $O_{1}$ geöffnet wird) sowie auf Intervalle geschah in bekannter Weise; die Nullstellung wurde sehr: häufig revidiert. Eine ganze Umdrehung der Schraube ver: ändert das Intervall $z$ wischen den Öffnungen beider Kontakte um 0,00019 sek. Der Knopf der Schraube ist in 100 Teile geteilt, so dass jeder dieser Teile 0,0000019 sek. bedeutet. Das Intervall, d. h. die Dauer der gestatteten Entladung, wurde zwischen 0,0001 und 0,005 Sek. $(0 ; 1-5 \sigma)$ gewählt $\left.{ }^{x}\right)$.

1) Es erscheint nicht überflüssig zu bemerken, dass man für die Entladung des übrig bleibenden Restes im Kondensator nicht zu sorgen braucht, da sich der Kondensator im Anfang jedes Einzelversuchs in einem bei $O_{2}$ (oder $T$ ) geschlossenen Kreise befindet. 
Für die unten zu erörternden Berechnungen musste der Widerstand des Entladungskreises bekannt sein; der Nerv wurde hierzu durch Umlegen einer Wippe in eine Wheatstone-Komhination aufgenommen; dies geschah am Anfang und am Ende der Versuchsreihe.

Jede Versuchsreihe verlief im übrigen so, dass für die eingestellte Entladungszeit eine nicht zu grosse Anzahl von Kapazitäten durchexperimentiert wurde, bald mit der grössten, bald mit der kleinsten beginnend, zuweilen auch nach Abschluss der Reihe in umgekehrter Folge zurückgehend. Für jede Kapazität wurde durch Stöpseln der Rheostaten $\alpha$ und $\beta$ das zur Minimalzuckung nötige Potential $p$ festgestellt; den Versuch, in welchem dies, $d . h$. die Minimalzuckung, grade erreicht ist, wollen wir als den "Grenzversuch" für die betr. Entladungszeit und Kapazität bezeichnen. Sind $\alpha$ und $\beta$ die Widerstände der gleichbezeichneten Rheostaten, $P$ die Spannung der Akkumulatorkette $A$ (meist. 2 oder 4 Volt), so ist

$$
p=\frac{\beta P}{\alpha+\beta} V_{0} \text { lt. }
$$

Da nach jedem Grenzversuch die zusammengehörigen Grössen $c, t, p$ und $w$ bekannt sind $(w$ ist die Summe des Nerven-, des etwaigen Zusatz- und des Galvanometerwiderstands, letzterer stets $=10000 \mathrm{Ohm}$ ), so kann man nach den oben angegebenen Formeln (1) leicht alle daselbst figurierenden Grössen berechnen, die mit * bezeichneten aber nur, falls die Selbstinduktion und Kapazität des Entladungskreises hinreichend klein sind.

Auf diesen letzteren, schon von $L$ a p i c qu e erörterten Punkt haben wir natürlich unsere volle Aufmerksamkeit gerichtet. Vor allem gibt es ein zuverlässiges, schon von Lapicque benutztes. Mittel, nachzusehen, wie weit der nach der obigen Formel berechnete Wert von $Q^{*}$ mit dem wirklichen übereinstimmt. Hierzu dient das Galvanometer $G$. Wir hatten dasselbe beständig mit im Kreise, was den Vorteil hat, es ohne jede Veränderung des Widerstandes, der Selbstinduktion usw. benutzen zu können ${ }^{1}$ ). Wir haben einfach

1) Lapicque schaltete, um das Verhältnis der wirksamen zur gesamten Quantität zu ermitteln, erst nach Abschluss der Versuchsreihe ein Galvano: meter ein, und verwendete, um grössere Ausschläge zu erhalten, ein höheres Potential als in den Zuckungsversuchen. Dass in diesen Versucben die am Galvanometer sich zeigende Quantität erheblich von der nach Formel (1) berechneten abwich, dürfte hauptsächlich der Selbstindultion und Kapazität des Galvanometers zuzuschreiben sein, die aber im eigentlichen Versuch fehlte. 
jeden Grenzversuch sofort wiederbolt, und zwar einmal bei offenem, đann bei geschlossenem $T$, hierbei aber statt des Präparats die Skala ins Auge gefasst. Nennt man die beiden Ausschläge $r$ und $s$, so ist offenbar auch mit Selbstinduktion usw. $r / s=Q^{*} / Q$, so dass man nur zu prüfen hat, $o b$

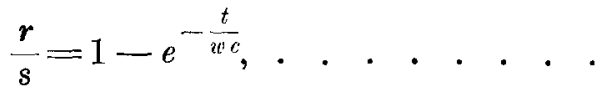

resp. welche Abweichungen vorhanden sind. Auf Grund des sehr unfangreichen Materials, uber das wir verfügen, können wir nun sagen, dass zwar fast stets $r / s$ etwas kleiner, zuweilen auch etwas grösser ist als der aus $c, t$ und $w$ berechnete Wert, dass aber die Abweichungen sich innerhalb weniger Prozente halten, so dass sie für die von uns aus den Versuchen gezogenen Schlüsse nicht wesentlich in Betracht kommen können. Ich bin sogar zu der Annabme geneigt, dass sie weniger auf wirklicher Selbstinduktion oder Kapazität, als auf Ungenauigkeiten (der Zeit- und Widerstandsbestimmung, der Ballistik usw.) beruhen. Diese Vermutung gründet sich vor allem darauf, dass in manchen Versuchsreihen so gut wie gar keine Abweichungen vorhanden waren. ferner die beobachteten $A b-$ weichungen in manchen Reihen durchweg entgegengesetzten Sinn hatten als in anderen, endlich keinerlei gesetzmässige Beziehung der Grösse der Abweichungen zu derjenigen der Kapazität oder der Entladungszeit hervortreten, wie es bei dem vorausgesetzten Ursprung hätte erwartet werden müssen.

Es ist auch nicht abzusehen, wo eine sehr erhebliche Selbstinduktion ihren Sitz haben sollte. Der Koëffizient des Drehspulengalvanometers, den ich schon in einigen ungefähren Auswertungen sehr klein gefunden hatte, beträgt nach einer freundlichen Mitteilung der Ursprungsfirma Siemens \& Halske etwa 0,02 Henry, d. h. $2 \cdot 10^{7} \mathrm{~cm}$. Das ist so wenig, dass es für unseren Gegenstand nicht in Betracht fällt. Nach den leicht aufstellbaren theoretischen Formeln ist nämlich ein erheblicher Einfluss einer Selbstinduktion vom Koëffizienten $q$ erst dann möglich, wenn $4 q / c$ nicht sehr klein ist gegen $w^{2}$. Bei uns war (s. oben) $w$ ohne Zusatzwiderstand, obwohl wir kurze Nervenstrecken vorzogen, mindestens $30000 \mathrm{Ohm}$ oder $3 \cdot 10^{13} \mathrm{~cm} / \mathrm{sek}$, und mit dem Zusatz $130000 \mathrm{Ohm}$ oder $13 \cdot 10^{13} \mathrm{~cm} / \mathrm{sek}$. Wie die folgende kleine Tabelle zeigt, ist selbst bei kleinen Kapazitäten $4 q / c$ verschwindend klein gegen $w^{2}$, sobald der Zusatzwiderstand im Kreise ist, und wird sogar ohne Zusatzwiderstand erst bei 0,001 Mf. einigermassen merklich. 


\begin{tabular}{|c|c|c|c|c|c|c|}
\hline \multirow{2}{*}{ in Mf. } & \multirow{2}{*}{$\begin{array}{c}\text { in } \\
\operatorname{sek}^{2} / \mathrm{cm}\end{array}$} & \multirow{2}{*}{$\begin{array}{c}4 q / \mathrm{c} \\
\text { in } \\
\mathrm{cm}^{2} / \mathrm{sek}^{2}\end{array}$} & \multicolumn{2}{|c|}{$w=30000 \quad 0 \mathrm{hm}$} & \multicolumn{2}{|c|}{$w=130000 \mathrm{Ohm}$} \\
\hline & & & $\begin{array}{c}w^{2} \text { in } \\
\mathrm{cm}^{2} / \mathrm{sek}^{2}\end{array}$ & $\begin{array}{l}\text { Verhältnis } \\
4 q / c: w^{2}\end{array}$ & $\begin{array}{c}w^{2} \text { in } \\
\mathrm{cm}^{2} / \mathrm{sek}^{2}\end{array}$ & $\begin{array}{l}\text { Verhältnis } \\
4 q / c: v v^{2}\end{array}$ \\
\hline $\begin{array}{l}1 \\
0,1 \\
0,01 \\
0,001\end{array}$ & $\begin{array}{l}10^{-15} \\
10^{-18} \\
10^{-17} \\
10^{-18}\end{array}$ & $\begin{array}{l}8 \cdot 10^{22} \\
8 \cdot 10^{23} \\
8 \cdot 10^{24} \\
8 \cdot 10^{25}\end{array}$ & $\begin{array}{l}9 \cdot 10^{26} \\
9 \cdot 10^{26} \\
9 \cdot 10^{26} \\
9 \cdot 10^{26}\end{array}$ & $\begin{array}{l}8,9 \cdot 10^{-5} \\
8,9 \cdot 10^{-4} \\
8,9 \cdot 10^{-3} \\
8,9 \cdot 10^{-2}\end{array}$ & $\begin{array}{l}169 \cdot 10^{26} \\
169 \cdot 10^{20} \\
169 \cdot 10^{26} \\
169 \cdot 10^{26}\end{array}$ & $\begin{array}{l}4,7 \cdot 10^{-6} \\
4,7 \cdot 10^{-5} \\
4,7 \cdot 10^{-4} \\
4,7 \cdot 10^{-3}\end{array}$ \\
\hline
\end{tabular}

Vollends kann von einem Einfluss einer etwaigen Selbstinduktion des Rheostaten, der noch dazu nur mit höchstens einigen Hundert Ohm im Kreise war, nicht die Rede sein. Auch die von mir nachgewiesene selbstinduktionsartige Eigenschaft des Nerven selbst kann keine Rolle spielen, weil sie nach aussen so gut wie wirkungslos ist.

Schwieriger ist es, über etwaige Kapazitätseinflüsse des Entladungskreises ins klare zu kommen, da die Kapazität eines Galvanometers, eines Rheostaten usw. weder bekannt oder bestimmbar, noch deren Einfluss theoretisch einfach zu übersehen ist ${ }^{1}$ ). Gegen die Wahrscheinlichkeit einer irgend erheblichen Einwirkung dieser Art spricht die schon oben ausgeführte Erwägung.

Wir haben übrigens nicht unterlassen, in besonderen Versuchen festzustellen, wieweit der Einfluss einer erheblichen und genau bekannten Selbstinduktion auf die in einer beschränkten Zeit entladene Elektrizitätsmenge merklich wird. In diesen Versuchen war der Nerv durch einen Rheostatwiderstand von gleicher Grössenordnung ersetzt. Mit densélben Werten von $t, c, p$, wie in irgendeinem Nervenversuch wurden dann die Ausschläge $r$ und $s$ abgelesen und nun das ganze wiederholt, nachdem eine Spule vom Koëffizienten $2 \cdot 10^{9} \mathrm{~cm}$ ohne Änderung des Gesamtwiderstandes in den Kreis aufgenommen

1) In einer früheren Arbeit (dieses Arch. Bd. 109 S. 95 ff. 1905) habe ich von einer Kapazität des Nerven gesprochen, da man bekanntlich rechnerisch die Polarisation wie die Ladung einer Kapazität behandeln kann. Dies ist, wie ich aus Referaten ersehe (Zentralbl. f. Physiol. Bd. 20 S. 565, Virchow's Jahresber. f. Anat. u. Physiol. 1906 S. 289, mein Jahresber. 1906 S. 43) von Cybulski \& Weissglas in einer mir unzugänglichen Arbeit vollkommon missverstanden worden. Die Verfasser finden die von mir beobachtete "Kapazität" mit Wechselströmen sehr klein, was ganz selbstverständlich ist; dass hinreichend frequente Wechselströme die Polarisation des Nerven teilweise beseitigen habe ich, was den Verfassern unbekannt zu sein scheint, schon 1872 und besonders' 1888 gefunden (vgl. dieses Arch. Bd. 5 S. 236 ff., Bd. 42 S. 39 ff.). 
war; und dann nochmals, nachdem ein Eisenkern in die Spule eingeführt worden war, durch den der Koëffizient auf etwa $2 \cdot 10^{10} \mathrm{~cm}$ erhöht wurde. In einigen solchen Reihen wurde auch die Selbstinduktion ohne Änderung des Widerstandes und der Kapazität dadurch variiert, dass die beiden Hälften eines Spulendrahtes einmal gleichsinnig und einmal gegensinnig geschaltet wurden ${ }^{1}$ ).

Diese Versuche ergaben nun, dass, solange nicht eine besonders grosse Selbstinduktion in den Kreis aufgenommen war, der Betrag $r$ 's ziemlich gut mit dem Wert von $1-e^{-\frac{t}{w c}}$ übereinstimmte, während mit Induktionsspulen der erstere hinter dem letzteren zurückblieb. Es wird genügen, ein einziges Versuchsbeispiel, und zwar von der letzterwähnten Art, anzuführen.

Im Entladungskreise die in der Anmerkung erwähnte Spule mit zwei Drahtabteilungen; der Widerstand des Entladungskreises (absichtlich klein genommen) ist $50000 \mathrm{Ohm}$. Entladungszeit $t=1,25 \sigma$.

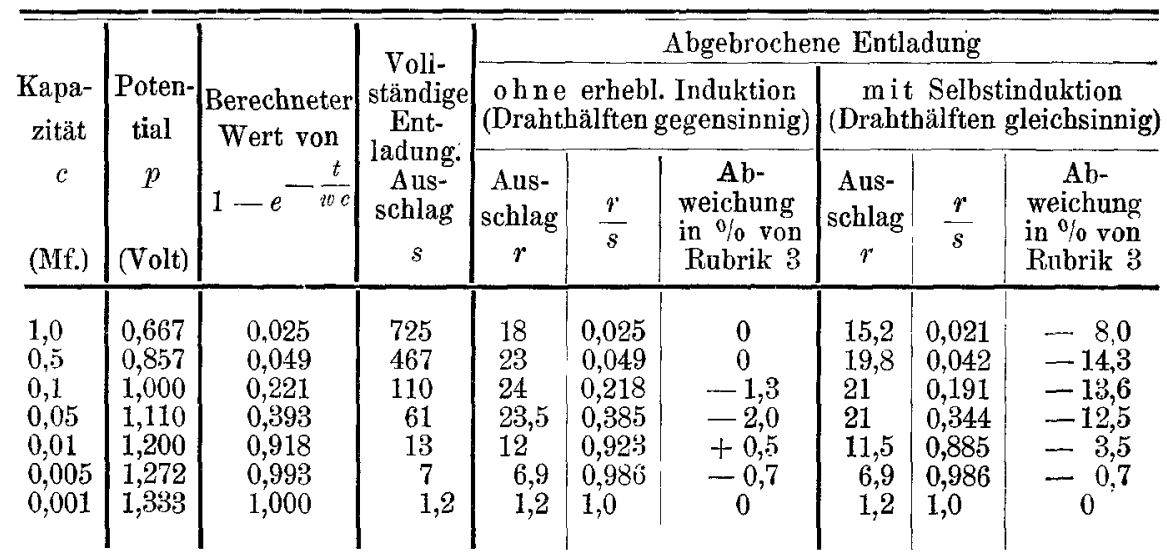

Der Versuch (und ebenso die übrigen) zeigt evident, dass selbst ohne hohen Widerstand die Selbstinduktion des Kreises erst dann in der Berechnung zu berücksichtigen ist, wenn absichtlich eine bohe Selbstinduktion eingeführt ist; ohne die letztere liegen die Einflüsse innerbalb der Fehlergrenzen.

Man darf also annehmen, dass, wenigstens mit grossem Zusatzwiderstand, die Selbstinduktion und Kapazität des Kreises die bei

1) $\mathrm{Zu}$ diesem letzteren Versuch diente eine doppelt gewickelte Galvanometerspule von $6900 \mathrm{Ohm}$ (Windungszahl 40000). Ihr Selbstinduktionskoëffizient wurde nicht bestimmt. 
abgebrochener Entladung abgegebene Quantität nicht merklich beeinflussen. Dann kann man aber auch die abgegebene Energie leicht berechnen; sie ist nämlich, wenn von den erörterten Einflüssen abgesehen werden darf, aus dem Ausdruck für $E^{*}$, Gleichung (1), zu entnehmen, in welchem alle Grössen bekannt sind. Da jedoch w während des Versuchs fast stets sich ändert, so ist es bei weitem vorzuziehen, sowohl $Q^{*}$ als $E^{*}$ mittels der Ausschläge $r$ und $s$ zu berechnen. Man erhält vämlich aus. Gleichung (2)

$$
\begin{gathered}
1-e^{-\frac{2 t}{2 v}}=\frac{r}{s}\left(2-\frac{r}{s}\right) . . . \\
\text { also } \quad . .
\end{gathered}
$$

Auf diese Weise sind in den unten (S. 185-191) folgenden Versuchen die Werte $Q^{*}$ und $E^{*}$ ermittelt.

Man erkennt leicht, dass die Genauigkeit der Berechnung dieser Grössen von der Genauigkeit abhängt, mit welcher $r$ und $s$ bestimmt sind. Von diesen Grössen ist $s$ hinreichend gut proportional den Werten $p c=Q$, wie man leicht feststellen kann, d. b. das Galvanometer ist hinreichend ballistisch. Die Werte von $r$ sind also schon an sich ein einwandfreies Mass für die abgegebene Quantität $Q^{*}$. Leider beträgt aber $r$ meist nur wenige Skalenteile trotz der hohen Empfindlichkeit des Galvanometers. Herr A d a m konnte mit Sicherheit noch Fünftel Skalenteile ablesen, so dass selbst die kleinsten $r$. Werte auf $10 \%$, die grösseren aber auf $5-2 \%$ zuverlässig sind. Für eine Anzahl von Versuchen wurden, um genauere $r$-Werte zu erlangen, am Schluss der Reihe die $r$-Ablesungen noch einmal mit einem erheblich grösseren Potential wienterholt und den so gewonnenen grösseren Zahlen proportional korrigiert. Ferner überschreiten bei den grössten Kapazitäten die s-Ablenkungen häufig den Skalenbereich; die so fehlenden $s$-Werte kann man aber leicht mittels der vorhandenen ergänzen, da ja die s-Werte den Quantitäten $Q$, welche durchweg bekannt sind, proportional sind. In den Versuchstabellen sind die genauesten $r / s$-Werte, die ermittelt werden konnten, verzeichnet.

Hinsichtlich der Zuverlässigkeit der Grössen $Q^{*}$ und $E^{*}$ scheint noch folgendes von Interesse. Von den beiden experimentell bestimmten Grössen $p$ und $r / s$ sei $p$ um $9 \%$ und $r / s$ um $\eta \%$ ungenau, und $\vartheta$ und $\eta$ seien kleine Zahlen; dann ist 
die prozentische Ungenauigkeit von $Q^{*}: \quad \boldsymbol{q}+\eta$,

$$
" \quad \quad \quad \quad \quad E^{*}: 2 \vartheta+2 \frac{1-r / s}{2-r / s} \eta .
$$

(Haben $\mathcal{y}$ und $\eta$ entgegengesetztes Vorzeichen, so können sich beide in gewissem Grade kompensieren.) Aus diesen Formeln folgt, dass $Q^{*}$ genauer ist als $E^{*}: 1$. wenn $\eta=0 ; 2$. wenn $r / s$ kleiner ist als etwa 0,5 . d. h. für die meisten Kapazitäten. Das Entgegengesetzte $\left(E^{*}\right.$ genauer als $\left.Q^{*}\right)$ kann nur eintreten, wenn $r / s$ sich 1 nähert (d. h. bei den kleinsten Kapazitäten zuweilen), weil nämlich $\eta$ wahrscheinlich in der Regel grösser sein wird als $\vartheta$. (Für $r / s=1$ liegt die betr. Grenze bei $\eta>\vartheta$, für $r / s=0,5$ bei $\eta>39$, für $r / s=0,1$ bei $\eta>19 \vartheta$ usw.)

\section{Versuche.}

1. Versuche mit A ufsuchung der Nutzzeit.

Von diesen, nach Lapicque's Verfahren angestellten Versuchen, welche dessen Resultat bestätigen, fübren wir nur ein Beispiel vollständig an, welches wegen des Umstandes von Interesse ist, dass es einen zehnmal so grossen Kapazitätsbereich umfasst als der eingangs angeführte Versuch.

Widerstand des Kreises 43000 Ohm (kein Zusatzwiderstand).

\begin{tabular}{c|c|c|c|c|c}
\hline Kapazität & $\begin{array}{c}\text { Erforderliches } \\
\text { Potential } \\
p\end{array}$ & $\begin{array}{c}\text { Erforderiche } \\
\text { Entladungszeit } \\
\text { (Nutzzeit) } \\
t\end{array}$ & \multicolumn{3}{|c|}{ Galvanometerausschiag } \\
(Mf.) & (Volt) & $\sigma$ & $s$ & $r$ & $\frac{r}{s}$ \\
\hline 0,001 & 1,429 & 0,108 & 2 & 2 & 1,0 \\
0,005 & 0,499 & 0,279 & 3. & 2,5 & 0,83 \\
0,01 & 0,374 & 0,374 & 4 & 3 & 0,75 \\
0,05 & 0,198 & 1,951 & 10 & 6 & 0,6 \\
0,1 & 0,186 & 2,198 & 20 & 7,5 & 0,375 \\
0,5 & 0,157 & 2,464 & 83 & 8,5 & 0,1024 \\
1,0 & 0,148 & 2,692 & 156 & 9 & 0,0577 \\
\end{tabular}

Die Fortsetzung dieser Tabelle (folgende Seite) hat sich der Leser nnmittelbar an die rechte seite der vorstehenden angefügt zu denken; die Tabelle konnte nicht auf die Breite einer Seite gebracht werden. 


\begin{tabular}{|c|c|c|c|c|c|c|}
\hline \multicolumn{7}{|c|}{ Berechnet } \\
\hline \multirow[b]{2}{*}{$\begin{array}{c}\text { Fnd- } \\
\text { potential } \\
p^{*} \\
\text { ber. aus } \\
w, t, c \\
\text { (Volt) }\end{array}$} & \multicolumn{3}{|c|}{ Elektrizitätsmenge } & \multicolumn{3}{|c|}{ Energie } \\
\hline & $\begin{array}{c}\text { vor- } \\
\text { handen } \\
Q \\
\text { (Hund }\end{array}$ & $\begin{array}{l}\text { abg } \\
\text { ber, aus } \\
w, t, c \\
\text { tstel Mik }\end{array}$ & ber. aus $\frac{r}{s}$ & $\begin{array}{l}\text { vor- } \\
\text { handen } \\
\quad E\end{array}$ & $\begin{array}{l}\text { abg } \\
\text { ber. aus } \\
w, t, c \\
\text { indertstel }\end{array}$ & $\begin{array}{l}\text { eben } \\
\text { ber.aus } \frac{\gamma}{s} \\
\text { rg) }\end{array}$ \\
\hline $\begin{array}{l}0,116 \\
0,138 \\
0,157 \\
0,080 \\
0,111 \\
0,136 \\
0,139\end{array}$ & $\begin{array}{r}0,14 \\
0,25 \\
0,37 \\
0,99 \\
1,86 \\
7,85 \\
14,80\end{array}$ & $\begin{array}{l}0,13 \\
0,18 \\
0,22 \\
0,59 \\
0,74 \\
0,85 \\
0,90\end{array}$ & $\begin{array}{l}0,14 \\
0,21 \\
0,28 \\
0,59 \\
0,70 \\
0,80 \\
0,85\end{array}$ & $\begin{array}{c}1,021 \\
0,622 \\
0,699 \\
0,980 \\
1,730 \\
6,16 \\
10,95\end{array}$ & $\begin{array}{l}1,014 \\
0,576 \\
0,577 \\
0,820 \\
1,107 \\
1,291 \\
1,289\end{array}$ & $\begin{array}{l}1,021 \\
0,605 \\
0,656 \\
0,823 \\
1,054 \\
1,197 \\
1,227\end{array}$ \\
\hline
\end{tabular}

Beiläufig bemerkt, zeigt sich hier, wie zu erwarten war, ein Minimum von $E$ (bei $0,005 \mathrm{Mf}$.). $p^{*}$ zeigt hier durchaus keine Konstanz (vgl. oben S. 174). Auch hier wird, wie die Vergleichung von $Q$ und $Q^{*}$, resp. $E$ und $E^{*}$ zeigt, ein um so kleinerer Teil der Quantität und der Energie verbraucht, je grösser die Kapazität: bei 0,001 Mf. fast alles, bei 1 Mf. von ersterer nur $1 / 16$, von letzterer nur 1/8. Während $Q$ im untersuchten Kapazitätsbereich bis auf mehr als das Hundertfache wächst, wäehst $Q^{*}$ zwar (wie bei La pi cque, s. oben S. 174) ebenfalls mit der Kapazität, aber nur auf das Siebenfache. Während $E$ auf das Elffache anwächst, ist bei $E^{*}$ höchstens eine Zunahme auf das 1,2 fache vorhanden; $E^{*}$ durchläuft ein Minimum bei etwa 0,005 Mf. Auch der Versuch von Lapicque zeigt ein solches (bei 0,02 Mf.). Dem entsprechen auch die übrigen Versuche. Alle zeigen auch ausnahmslos, dass $E$ ein Minimum durchläuft, und dass das Minimum von $E^{*}$ häufig mit dem von $E$ zusammentrifft.

Erwähnt sei noch, dass auch in diesem Versuch der aus $r$ und $s$ und der aus der Exponentialfunktion berechnete Wert von $Q^{*}$, ebenso von $E^{*}$, recht gut übereinstimmen, ein Beweis, dass die Selbst-. induktion und die Kapazität des Entladungskreises, abweichend von den Umständen bei Lapicque, keine wesentliche Rolle spielten.

Die übrigen Versuche dieser Art sollen nur dazu verwendet werden, eine Zusammenstellung der gefundenen Nutzzeiten (mit Einschluss der beiden bereits vorliegenden Reihen) zu geben: 


\begin{tabular}{|c|c|c|c|c|c|c|c|c|c|}
\hline & \multicolumn{7}{|c|}{ Kapazitäten in Mf. } & \multirow{2}{*}{$\begin{array}{l}\text { Ungefährer } \\
\text { Widerstand } \\
(0 \mathrm{hm})\end{array}$} & \multirow{2}{*}{ Versuch von } \\
\hline & 1,0 & 0,5 & 0,1 & 0,05 & 0,01 & 0,005 & 0,001 & & \\
\hline $\begin{array}{c}\text { Ge- } \\
\text { fundene } \\
\text { Nutz- } \\
\text { zeiten } \\
\text { in } \sigma\end{array}$ & $\left\{\begin{array}{l}1,81 \\
2,692 \\
2,0 \\
2,01 \\
2,22 \\
2,732 \\
4,63 \\
4,917\end{array}\right.$ & $\begin{array}{l}-\overline{2} \\
1,464 \\
1,06 \\
- \\
- \\
-\end{array}$ & $\begin{array}{l}1,54 \\
2,198 \\
0,68 \\
0,94 \\
1,38 \\
2,447 \\
3,35 \\
4,157\end{array}$ & $\begin{array}{l}1,35 \\
1,951 \\
0,49 \\
0,83 \\
= \\
= \\
-\end{array}$ & $\begin{array}{l}0,63 \\
0,374 \\
0,3 \\
0,26 \\
0,87 \\
0,927 \\
1,95 \\
2,276\end{array}$ & $\begin{array}{l}\overline{-} \\
0,279 \\
0,2 \\
0,29 \\
- \\
- \\
-\end{array}$ & $\begin{array}{l}-\overline{108} \\
0,1 \\
\overline{0,22} \\
0,452 \\
0,59 \\
0,642\end{array}$ & $\begin{array}{r}70000 \\
43000 \\
28000 \\
28000 \\
43500 \\
133400 \\
140000 \\
147200\end{array}$ & $\begin{array}{c}\text { Lapicque } \\
\text { Adam (s. ob.) } \\
\text { Ad am } \\
" \\
" \\
" \\
"\end{array}$ \\
\hline
\end{tabular}

Man erkennt aus diesen Zahlen, dass die Nutzzeit für eine gegebene Kapazität sehr erheblich bei verschiedenen Präparaten, sicher auch nach Widerstand und Streckenlänge variiert. Endlich sei noch folgendes bemerkt: Stellt man die Nutzzeiten als Ordinaten einer Kurve dar, deren Abszissen die Kapazitäten sind, so zeigt sich, dass die Nutzzeit von der grössten Kapazität ab anfangs kaum merklich, und erst bei den kleinsten Kapazitäten sehr entschieden sinkt. Mit anderen Worten: Im grössten Teil der Kapazitäten ist die Nutzzeit merklich konstant und von derjenigen Grössenordnung, wie sie sich aus meinen früheren Versuchen als wahrscheinlich ergeben hat ${ }^{1}$ ).

2. Versuche mit gegebener Entladungszeit.

Um dem Leser ein völlig unbeeinflusstes Urteil zu ermöglichen, führen wir sämtlich e überhaupt angestellten Versuchsreihen dieser Art an, und unterdrücken auch keinen Finzelversuch, auch wenn er uns aus irgendwelchen Gründen verdächtig schien. Wo eine Angabe für 0,001 Mf. fehlt, liegt der Grund nur darin, dass bei der kleinsten Kapazität keine Zuckung zu erhalten war. In den Widerstandsangaben bedeutet die erste Zahl den Widerstand der Nervenstrecke, die zweite den des Galvanometers, die dritte den etwaigen Zusatz.

1. Entladungszeit $\mathbf{0 , 1}$ o. $100000 \Omega$ Zusatz.

\begin{tabular}{|c|c|c|c|c|c|c|}
\hline \multirow{2}{*}{$\begin{array}{c}c \\
\text { Mf. }\end{array}$} & \multirow{2}{*}{$\begin{array}{c}p \\
\text { Volt }\end{array}$} & \multirow{2}{*}{$\frac{r}{s}$} & $Q$ & $Q^{*}$ & $E$ & $E^{*}$ \\
\hline & & & \multicolumn{2}{|c|}{ Hundertstel Mikrocoul. } & \multicolumn{2}{|c|}{ Hundertstel Erg } \\
\hline 1,0 & 2,122 & 0,00111 & 212,2 & 0,236 & 2252 & 5,002 \\
\hline 0,5 & 2,182 & 0,00216 & 109,1 & $\mathbf{0 , 2 3 6}$ & 1190 & 5,137 \\
\hline 0,1 & 2,206 & 0,01064 & 22,1 & 0,285 & 243,4 & $\mathbf{5 , 1 5}$ \\
\hline 0,05 & 2,182 & 0,02174 & 10,9 & 0,287 & $119^{\circ}$ & 5,117 \\
\hline 0,01 & 2,214 & 0,1053 & 2,2 & $\mathbf{0}, \mathbf{2 g 3}$ & 24,5 & 4,890 \\
\hline 0,005 & 2,400 & 0,2 & 1,2 & 0.240 & 14,4 & 5,184 \\
\hline
\end{tabular}

1) Hierdurch wird es auch verständlich, dass in meiner letzten Arbeit die darin enthaltene Annahme ziemlich gut sich zu bewähren schien. 


\begin{tabular}{|c|c|c|c|c|c|c|}
\hline$c$ & $p$ & $\frac{r}{s}$ & $Q$ & $Q^{*}$ & $E$ & $E^{*}$ \\
\hline
\end{tabular}

2. Entladungszeit $0,1 \sigma . \quad w=31100+10000+100000=141100 \Omega$.

\begin{tabular}{|c|c|c|c|c|c|c|}
\hline 1,0 & 2,411 & 0,00102 & 241,1 & 0,243 & 2905 & 5,878 \\
\hline 0,5 & 2,509 & 0,00195 & 125,4 & 0,244 & 1574 & 6,120 \\
\hline 0,1 & 2,681 & 0,00909 & 26,8 & 0,244 & 359,5 & 6.506 \\
\hline 0,05 & 2,824 & 0,01739 & 14,1 & 0,245 & 199,3 & 6,872 \\
\hline 0.01 & 3,321 & 0,07407 & 3,3 & 0,216 & 55,1 & 7,867 \\
\hline 0,005 & 3,442 & 0,1429 & 1,7 & 0,216 & 29,6 & 7,857 \\
\hline 0,001 & 4,868 & 0,5 & 0,5 & 0,243 & $11 ; 9$ & 8,886 \\
\hline
\end{tabular}

3. Entladungszeit 0,1 $\sigma .100000 \Omega$ Zusatz.

\begin{tabular}{l|l|l|l|l|r|r}
\hline 0,001 & 3,333 & 0,5 & 0,33 & $\mathbf{0 , 1 6 7}$ & 5,56 & $\mathbf{4 , 1 6 7}$ \\
0,005 & 2,728 & 0,1818 & 1,36 & $\mathbf{0 , 2 4 9}$ & 18,6 & $\mathbf{6 , 1 5 0}$ \\
0,01 & 2,485 & 0,0952 & 2,49 & $\mathbf{0 , 2 3 7}$ & 30,87 & $\mathbf{5 , 6 0 1}$ \\
0,05 & 2,462 & 0,0198 & 12,31 & $\mathbf{0 , 2 4 4}$ & 151,5 & $\mathbf{5 , 9 4 0}$ \\
0,1 & 2,444 & 0,01005 & $\mathbf{2 4 , 4 4}$ & $\mathbf{0 , 2 4 6}$ & 298,6 & $\mathbf{5 , 9 7 1}$ \\
0,5 & 2,462 & 0,00198 & $\mathbf{1 2 3 , 1}$ & $\mathbf{0 , 2 4 3}$ & $\mathbf{1 5 1 5}$ & $\mathbf{5 , 9 8 6}$ \\
1,0 & 2,467 & 0,00099 & 246,7 & $\mathbf{0 , 2 4 3}$ & 3044 & $\mathbf{6 , 0 0 3}$
\end{tabular}

4. Entladungszeit $\mathbf{0 , 1} \sigma . \quad w=33100+10000+100000=143100 \Omega$.

\begin{tabular}{l|l|l|r|r|r|r}
\hline 1,0 & 2,502 & 0,00049 & 250,2 & $\mathbf{0 , 1 2 2}$ & 3130,5 & $\mathbf{3 , 0 5 0}$ \\
0,1 & 2,513 & 0,00728 & 25,13 & $\mathbf{0 , 1 8 3}$ & 315,8 & $\mathbf{4 , 5 8 9}$ \\
0,01 & 2,616 & 0,06818 & 2,62 & $\mathbf{0 , 1 7 8}$ & 34,2 & $\mathbf{4 , 5 0 7}$ \\
0,001 & 3,748 & 0,4667 & 0,37 & $\mathbf{0 , 1 7 5}$ & 7,03 & $\mathbf{5 , 0 2 7}$
\end{tabular}

5. Entladungezeit 0,1 $\circ .100000 \Omega$ Zusatz.

\begin{tabular}{l|c|c|c|c|c|c}
\hline 1,0 & 2,222 & 0,00107 & 222,2 & $\mathbf{0 , 2 3 8}$ & 2469 & $\mathbf{5 , 2 8 4}$ \\
0,5 & 2,316 & 0,00205 & 115,8 & $\mathbf{0 , 2 3 8}$ & 1341 & $\mathbf{5 , 9 0 3}$ \\
0,1 & 2,367 & 0,01005 & 23,7 & $\mathbf{0 , 2 3 8}$ & 280,2 & $\mathbf{5 , 6 0 4}$ \\
0,05 & 2,431 & 0,01963 & 12,16 & $\mathbf{0 , 2 3 8}$ & $\mathbf{1 4 7}, 8$ & $\mathbf{5 , 7 3 3}$ \\
0,01 & 2,597 & 0,09091 & 2,6 & $\mathbf{0 , 2 3 6}$ & 33,7 & $\mathbf{5 , 8 5 0}$ \\
0,005 & 2,797 & 0,1667 & 1,4 & $\mathbf{0 , 2 3 3}$ & 19,56 & $\mathbf{5 , 9 7 5}$ \\
0,001 & $\mathbf{4}, 235$ & $\mathbf{0 , 5 7 1 4}$ & 0,42 & $\mathbf{0 , 2 4 2}$ & 8,97 & $\mathbf{7 , 3 2 4}$ \\
\hline
\end{tabular}

6. Entlaringszeit 0,1 $\sigma .100000 \Omega$ Zusatz.

\begin{tabular}{l|l|l|r|r|r|r}
\hline 1,0 & 1,403 & 0,001 & 140,3 & $\mathbf{0 , 1 4 0}$ & 983,6 & $\mathbf{1 , 9 6 3}$ \\
0,001 & 2,214 & 0,6 & 0,22 & $\mathbf{0 , 1 3 3}$ & 2,45 & $\mathbf{2 , 0 5 9}$ \\
0,5 & 1,561 & 0,00179 & 78,05 & $\mathbf{0 , 1 4 0}$ & 609,2 & $\mathbf{2 , 1 8 3}$ \\
0,005 & 1,838 & 0,15 & 0,92 & $\mathbf{0 , 1 3 3}$ & 8,45 & $\mathbf{2 , 3 4 3}$ \\
0,1 & 1,714 & 0,00811 & 17,14 & $\mathbf{0 , 1 3 9}$ & 146,9 & $\mathbf{2 , 3 7 3}$ \\
0,01 & 1,814 & 0,08 & 1,81 & $\mathbf{0 , 1 4 5}$ & 16,46 & $\mathbf{2 , 4 2 8}$ \\
0,05 & 1,790 & 0,01579 & 8,95 & $\mathbf{0 , 1 4 1}$ & 81,97 & $\mathbf{2 , 5 6 8}$ \\
\hline
\end{tabular}

7. ${ }^{1)}$ Entladungszeit 0,1 $\sigma .100000 \Omega$ Zusatz.

\begin{tabular}{l|l|l|l|l|l|l}
\hline 1,0 & 1,895 & 0,00076 & 189,5 & $\mathbf{0 , 1 3 7}$ & 1795 & $\mathbf{2 , 5 9 7}$ \\
0,1 & 1,97 & 0,00702 & 19,7 & $\mathbf{0 , 1 3 8}$ & 194 & $\mathbf{2 , 7 1 3}$ \\
0,01 & 2,113 & 0,06667 & 2,11 & $\mathbf{0 , 1 4 1}$ & 22,93 & $\mathbf{2 , 8 7 7}$ \\
0,001 & 3,005 & 0,48 & 0,3 & $\mathbf{0 , 1 4 4}$ & 4,52 & $\mathbf{3 , 2 9 4}$
\end{tabular}

1) In unmittelbarem Anschluss an Versuch 6, und an demselben Präparat angestellt. 


\begin{tabular}{|c|c|c|c|c|c|c|}
\hline$c$ & $p$ & $\frac{r}{s}$ & $Q$ & $Q^{*}$ & $\dot{E}$ & $E^{*}$ \\
\hline
\end{tabular}

8. Entladungszeit $0,1 \quad \sigma .100000 \Omega$ Zusatz.

\begin{tabular}{|c|c|c|c|c|c|c|}
\hline 1,0 & 1,260 & 0,00107 & 126 & $0,1+5$ & 794,2 & 1,699 \\
\hline 0,8 & 1,351 & 0,00125 & 108,1 & 0,135 & 730,1 & 1,821 \\
\hline 0,6 & 1,484 & 0,00151 & 89,06 & 0,133 & 660,9 & 2,000 \\
\hline 0,4 & 1,546 & 0,00212 & 61,84 & 0,131 & 478,0 & $\mathbf{2 , 0 2 2}$ \\
\hline 0,2 & 1,647 & 0,00405 & 32,94 & 0,139 & 271,3 & 2,149 \\
\hline 0,1 & 1,674 & 0,00810 & 16.74 & 0,136 & 140,2 & $\mathbf{2}, 262$ \\
\hline 0,08 & 1,740 & 0,00980 & 13,92 & 0,136 & 121,1 & 2,363 \\
\hline 0,06 & 1.802 & 0,01268 & 10,81 & 0,137 & 97,44 & 2,455 \\
\hline 0,04 & 1,895 & 0,01796 & 7,58 & 0,136 & 71.8 & 2,554 \\
\hline 0,02 & 1,938 & 0,03535 & 388 & 0,137 & 37,56 & $\mathbf{2 , 6 0 9}$ \\
\hline 0,01 & 2,030 & 0,07353 & 2,03 & 0,149 & 20,6 & 2,917 \\
\hline
\end{tabular}

9. Entladungszeit $\mathbf{0 , 1}$ \%. $100000 \Omega$ Zusatz.

\begin{tabular}{l|l|l|l|l|r|r}
\hline 0,01 & $\mathbf{1}, 484$ & 0,08974 & 1,484 & $\mathbf{0 , 1 3 3}$ & 11,02 & $\mathbf{1 , 8 8 8}$ \\
0,008 & $\mathbf{1 , 5 1 6}$ & 0,1167 & 1,212 & $\mathbf{0 , 1 4 1}$ & 9,19 & $\mathbf{2 , 0 1 9}$ \\
0,006 & 1,619 & 0,1458 & 0,971 & $\mathbf{0 . 1 4 2}$ & 7,86 & $\mathbf{2 , 1 2 6}$ \\
0,004 & $\mathbf{1 , 7 1 4}$ & 0,1944 & 0,686 & $\mathbf{0 , 1 3 3}$ & 5,88 & $\mathbf{2 , 0 6 1}$ \\
0,002 & $\mathbf{1}, 990$ & 0,3601 & 0,398 & $\mathbf{0 , 1 4 3}$ & 3,96 & $\mathbf{2 , 3 3 8}$ \\
0,001 & 2,519 & 0,5833 & 0,252 & $\mathbf{0 , 1 4 7}$ & 3,17 & $\mathbf{2 , 6 2 1}$
\end{tabular}

10. Entladungszeit 0,1 $\sigma . \quad v=23500+10000+100000=133500 \Omega$.

\begin{tabular}{l|l|l|r|r|r|r}
\hline 0,001 & 3,385 & 0,5 & 0,339 & $\mathbf{0 , 1 6 9}$ & 5,73 & $\mathbf{4 , 2 9 8}$ \\
0,002 & 2,933 & 0,3333 & 0,587 & $\mathbf{0 , 1 9 6}$ & 8,60 & $\mathbf{4 , 7 8 0}$ \\
0,004 & 2,621 & $0, \mathbf{1 8 9 4}$ & $\mathbf{1 , 0 4 8}$ & $\mathbf{0 , 1 9 8}$ & 13,74 & $\mathbf{4 , 7 1 0}$ \\
0,006 & 2,680 & 0,1235 & 1,608 & $\mathbf{0 , 1 9 8}$ & 21,55 & $\mathbf{4 , 9 9 2}$ \\
0,008 & 2,693 & 0,0926 & 2,154 & $\mathbf{0 , 1 9 9}$ & 29,01 & $\mathbf{5 , 1 2 8}$ \\
0,01 & 2,671 & 0,030 & 2,671 & $\mathbf{0 , 2 1 4}$ & 35,67 & $\mathbf{5 , 4 8 0}$ \\
0,02 & 2,644 & 0,040 & 5,287 & $\mathbf{0 , 2 1 1}$ & 69,91 & $\mathbf{5 , 4 8 1}$ \\
0,04 & 2,769 & 0,0215 & 11,08 & $\mathbf{0 , 2 3 8}$ & 153,40 & $\mathbf{6 , 5 2 6}$ \\
0,06 & 2,758 & 0,01439 & 16,55 & $\mathbf{0 , 2 3 8}$ & 228,10 & $\mathbf{6 , 5 1 8}$ \\
0,08 & 2,802 & 0,01058 & 22,42 & $\mathbf{0 , 2 3 7}$ & 314,10 & $\mathbf{6 , 6 1 3}$ \\
0,1 & 2,880 & 0,00823 & 28,80 & $\mathbf{0 , 2 3 7}$ & 414,60 & $\mathbf{6 , 7 9 6}$ \\
0,5 & 2,987 & 0,00159 & 149,40 & $\mathbf{0 , 2 3 7}$ & 2231,0 & $\mathbf{7 , 0 7 9}$ \\
1,0 & 3,024 & 0,00078 & 302,40 & $\mathbf{0 , 2 3 7}$ & 4573,50 & $\mathbf{7 , 1 6 9}$ \\
\hline
\end{tabular}

11. Entladungszeit 0,1 $\sigma . w=64000+10000+100000=174000 \Omega$.

\begin{tabular}{|c|c|c|c|c|c|c|}
\hline 0,001 & 2,876 & 0,40 & 0,288 & 0,115 & 4,14 & $\mathbf{2 , 6 4 8}$ \\
\hline 0,003 & 2,361 & 0,1667 & 0,708 & 0,118 & 8,36 & 2,554 \\
\hline 0,005 & 1,860 & 0.1250 & 0,981 & 0,116 & 8,66 & 2,029 \\
\hline 0,01 & 1,674 & 0,0714 & 1,674 & 0,120 & 14,02 & $\overrightarrow{1}, 931$ \\
\hline 0,02 & 1,590 & 0,0371 & 3,181 & 0,118 & 25,29 & $\mathbf{1}, \mathbf{8 3 9}$ \\
\hline 0,04 & 1,576 & 0,01887 & 6,303 & 0,119 & 49,66 & $\mathbf{1}, \mathbf{5 5 6}$ \\
\hline 0,08 & 1,576 & 0,00935 & 12,610 & 0,118 & 99,32 & 1,848 \\
\hline 0,15 & 1,590 & 0,0050 & 23,855 & 0,119 & 189,70 & 1,842 \\
\hline 0,3 & 1,633 & 0,00238 & 48,990 & 0,117 & 400,10 & 1,903 \\
\hline 0,5 & 1,619 & 0,00146 & 80,950 & 0,118 & 655.30 & 1,406 \\
\hline 1,0 & 1,647 & 0,00072 & 164,70 & 0,118 & 1356,0 & $1, \$ 41$ \\
\hline 0,001 & 2,268 & 0,50 & 0,227 & $\mathbf{0 , 1 1 3}$ & 2,57 & 1,930 \\
\hline 0,005 & 1,753 & 0,1429 & 0,876 & 0,125 & 7,68 & $\mathbf{2}, 038$ \\
\hline 0,08 & ${ }^{*} 1,688$ & 0,0090 & 13,50 & 0,122 & 11,40 & 2,044 \\
\hline
\end{tabular}




\begin{tabular}{l|l|l|l|l|l|l|l|l|l}
\hline & $p$ & $\frac{r}{s}$ & $Q$ & $Q^{*}$ & $E^{*}$ \\
\hline
\end{tabular}

12. Entladungszeit 0,1 o. $w=19600+10000+100000=129600 \Omega$.

\begin{tabular}{l|l|l|r|r|r|r}
\hline 1,0 & 1,869 & 0,00107 & 186,90 & $\mathbf{0 , 2 0}$ & 1746,60 & $\mathbf{9 , 7 4 0}$ \\
0,5 & 2,222 & 0,0018 & 111,10 & $\mathbf{0 , 2 0}$ & 1234,30 & $\mathbf{4 , 4 4 2}$ \\
0,1 & 2,316 & 0,0107 & 23,20 & $\mathbf{0 , 2 5}$ & 268,15 & $\mathbf{5 , 7 0 8}$ \\
0,05 & 2,476 & 0,0202 & 12,40 & $\mathbf{0 , 2 5}$ & 153,30 & $\mathbf{6 , 1 3 6}$ \\
0,01 & 2,571 & 0,096 & 2,60 & $\mathbf{0 , 9 4 7}$ & 33,06 & $\mathbf{6 , 0 1 3}$ \\
0,005 & 2,740 & 0,18 & 1,40 & $\mathbf{0 , 9 4 7}$ & 18,77 & $\mathbf{6 , 1 5}$ \\
0,001 & 3,848 & 0,5 & 0,39 & $\mathbf{0 , 1 9 2}$ & 7,74 & $\mathbf{5 , 5 5}$ \\
\hline
\end{tabular}

13. Entladungszeit 0,2 o. $w=35100+10000+100000=145100 \Omega$.

\begin{tabular}{l|l|l|r|r|r|r}
1,0 & 0,6942 & 0,00341 & 69,42 & $\mathbf{0 , 2 8 7}$ & 241,0 & $\mathbf{1 , 6 4 0}$ \\
0,5 & 0,8254 & 0,00451 & 41,27 & $\mathbf{0 , 1 8 6}$ & 170,30 & $\mathbf{1 , 5 3 1}$ \\
0,1 & 0,8750 & 0,02083 & 8,75 & $\mathbf{0 , 1 8 2}$ & 38,28 & $\mathbf{1 , 5 7 8}$ \\
0,05 & 0,8992 & 0,04054 & 4,496 & $\mathbf{0 , 1 8 2}$ & 20,22 & $\mathbf{1 , 6 0 6}$ \\
0,01 & 1,0150 & 0,1875 & 1,015 & $\mathbf{0 , 1 9 0}$ & 5,15 & $\mathbf{1 , 7 5 0}$ \\
0,005 & 1,1430 & 0,30 & 0,571 & $\mathbf{0 , 1 7 1}$ & 3,27 & $\mathbf{1 , 6 6 5}$ \\
0,001 & 1,9270 & 0,70 & 0,193 & $\mathbf{0 , 1 3 5}$ & 1,86 & $\mathbf{1 , 6 4 0}$
\end{tabular}

14. Entladungszeit 0,2 o. $100000 \Omega$ Zusatz.

\begin{tabular}{l|l|l|r|r|r|r}
\hline 1,0 & 0,6527 & 0,00173 & 65,27 & $\mathbf{0 , 1 1 3}$ & 213,0 & $\mathbf{0 , 7 8 5}$ \\
$\mathbf{0 , 5}$ & 0,7078 & 0,00327 & 35,39 & $\mathbf{0 , 1 1 6}$ & 125,30 & $\mathbf{0 , 8 1 7}$ \\
0,1 & 07213 & 0,01640 & 7,213 & $\mathbf{0 , 1 1 8}$ & 26,01 & $\mathbf{0 , 8 1 6}$ \\
0,05 & 0,7347 & 0,03226 & $\mathbf{3 , 6 7 3}$ & $\mathbf{0 , 1 1 8}$ & $\mathbf{1 3 , 5 0}$ & $\mathbf{0 , 8 5 7}$ \\
0,01 & 0,8254 & 0,1426 & 0,825 & $\mathbf{0 , 1 1 8}$ & 3,41 & $\mathbf{0 , 9 0 4}$ \\
0,005 & 0,8992 & 0,2857 & 0,450 & $\mathbf{0 , 1 2 8}$ & 2,07 & $\mathbf{1 , 0 1 3}$ \\
0,001 & 1,4530 & 1,0 & 0,145 & $\mathbf{0 , 1 4 5}$ & 1,05 & $\mathbf{1 , 0 5 5}$
\end{tabular}

15. Entladungszeit 0,2 $\sigma . \quad w=11100+10000+100000=121100 \Omega$

\begin{tabular}{l|l|l|l|l|l|l}
\hline 1,0 & $\mathbf{2}, 841$ & 0,00171 & 284,1 & $\mathbf{0 , 4 8 6}$ & 4034,5 & $\mathbf{1 3 , 7 9}$ \\
0,5 & $\mathbf{2 , 8 2 4}$ & 0,00344 & 141,2 & $\mathbf{0 , 4 5 6}$ & 1993 & $\mathbf{1 3 , 6 9}$ \\
0,1 & 2,881 & 0,0167 & 28,81 & $\mathbf{0 , 4 8 1}$ & 415,05 & $\mathbf{1 3 , 7 5}$ \\
0,05 & 2,974 & 0,033 & 14,87 & $\mathbf{0 , 4 9 1}$ & 221,15 & $\mathbf{1 4 , 3 6}$ \\
0,01 & 3,249 & 0,147 & 3,25 & $\mathbf{0 , 4 7 8}$ & 53 & $\mathbf{1 4 , 4 4}$ \\
0,005 & 3,548 & 0,29 & 1,77 & $\mathbf{0 , 5 1 5}$ & 31,47 & $\mathbf{1 5 , 5 9}$
\end{tabular}

16. Entladungszeit 0,45 $\sigma . \quad w=41300+10000+100000=151300 \Omega$.

\begin{tabular}{|c|c|c|c|c|c|c|}
\hline 1,0 & 1,445 & 0,00326 & 144,5 & 0,470 & 1043,5 & 6,79 \\
\hline 0,5 & 1,445 & 0,00651 & 72,25 & 0,451 & 522 & 6,78 \\
\hline 0,1 & 1,445 & 0,032 & 14,45 & 0,463 & 104,4 & 6,57 \\
\hline 0,05 & 1,448 & 0,065 & 7,24 & 0,471 & 52,5 & 6,09 \\
\hline 0,01 & 1,602 & 0,3 & 1,6 & 0,481 & 12,84 & 6,54 \\
\hline 0,005 & 1,820 & 0,45 & 0,91 & 0,410 & 8,28 & 5,78 \\
\hline 0,001 & 3,339 & 1,0 & 0,334 & 0,834 & 5,58 & 5,58 \\
\hline
\end{tabular}

17. Entladungszeit 0,5 o. $w=41400+10000+100000=151400 \Omega$.

\begin{tabular}{l|l|l|c|c|c|c}
1,0 & 1,463 & 0,00824 & 146,3 & $\mathbf{0 , 4 7 4}$ & 1070,5 & $\mathbf{6 , 9 3}$ \\
0,5 & 1,529 & 0,00620 & 76,45 & $\mathbf{0 , 4 7 4}$ & 585 & $\mathbf{7 , 2 3}$ \\
0,2 & 1,561 & 0,0151 & 31,22 & $\mathbf{0 , 4 7 1}$ & 243,5 & $\mathbf{7 , 3 0}$ \\
0,1 & 1,646 & 0,0288 & 16,46 & $\mathbf{0 , 4 7 4}$ & 125,5 & $\mathbf{7 , 6 9}$ \\
0,05 & 1,684 & 0,0568 & 8,42 & $\mathbf{0 , 4 7 8}$ & 70,9 & $\mathbf{7 , 8 3}$ \\
0,02 & 1,789 & 0,131 & 3,58 & $\mathbf{0 , 4 6 3}$ & 32 & $\mathbf{7 , 8 4}$ \\
0,01 & 1,922 & 0,262 & 1,92 & $\mathbf{0 , 5 1 5}$ & 18,47 & $\mathbf{8 , 4 1}$ \\
0,005 & 2,222 & 0,438 & 1,11 & $\mathbf{0 , 4 8 6}$ & 12,35 & $\mathbf{8 , 4 4}$ \\
0,002 & 2,968 & 0,714 & 0,59 & $\mathbf{0 , 4 2 1}$ & 8,8 & $\mathbf{8 , 0 9}$
\end{tabular}




\begin{tabular}{l|l|l|l|l|l|l|l}
\hline & $p$ & $\frac{r}{s}$ & $Q$ & $Q^{*}$ & $E$ & $E^{*}$ \\
\hline
\end{tabular}

18. Entladungszeit 0,75 $\sigma . \quad w=25000+10000=35000 \Omega$.

\begin{tabular}{l|l|l|l|l|l|l}
\hline 0,005 & 1,193 & 1,0 & 0,597 & $\mathbf{0 , 5 9 7}$ & 3,56 & $\mathbf{3 , 5 6}$ \\
0,01 & 0,824 & 0,89 & 0,824 & $\mathbf{0 , 7 2 3}$ & $\mathbf{3 , 3 9}$ & $\mathbf{3}, \mathbf{3 5}$ \\
0,05 & 0,476 & 0,38 & 2,38 & $\mathbf{0 , 9 0 5}$ & 5,67 & $\mathbf{3}, \mathbf{4 9}$ \\
0,1 & 0,428 & 0,216 & 4,28 & $\mathbf{0 , 9 2 5}$ & 9,17 & $\mathbf{3 , 5 1}$ \\
$0, \mathbf{5}$ & 0,394 & 0,044 & $\mathbf{1 9}$ & $\mathbf{0 , 8 6 8}$ & 38,73 & $\mathbf{3 , 3 4}$ \\
$\mathbf{1 , 0}$ & 0,394 & 0,0224 & 39,4 & $\mathbf{0 , 8 6 6}$ & 77,45 & $\mathbf{3 , 4 3}$
\end{tabular}

19. Entladungszeit 0,75 $\sigma . w=92600+10000=102600 \Omega$. Lange Nervenstrecke.

\begin{tabular}{|c|c|c|c|c|c|c|}
\hline 0,001 & 1,266 & 1,0 & 0,127 & 0,127 & 0,8 & 0,8 \\
\hline 0,005 & 0,453 & 0,8 & 0,227 & 0,181 & 0,51 & 0,499 \\
\hline 0,01 & 0,337 & 0,57 & 0,337 & 0,142 & 0,57 & $0, \mathbf{4 6 5}$ \\
\hline 0,05 & 0,268 & 0,14 & 1,34 & $\mathbf{0 , 1 8 8}$ & 1,8 & 0,478 \\
\hline 0,1 & 0,246 & 0,08 & 2,46 & 0,197 & 3,02 & $\mathbf{0 , 4 6 4}$ \\
\hline 0,5 & 0,230 & 0,0169 & 11,5 & 0,194 & 13,24 & $\mathbf{0 , 4 4 5}$ \\
\hline 1,0 & 0,230 & 0,0084 & 23,0 & 0,143 & 26,47 & $0, \mathbf{4 1 7}$ \\
\hline
\end{tabular}

20. Entladungszeit 0,75 $\alpha . w=111700+10000=121700 \Omega$. Lange Nervenstrecke.

\begin{tabular}{l|l|l|l|l|l|l}
\hline 0,001 & $\mathbf{1}, 529$ & 1,0 & 0,153 & $\mathbf{0 , 1 5 3}$ & 1,17 & $\mathbf{1 , 1 7}$ \\
$\mathbf{0}, 005$ & 0,566 & 0,66 & $\mathbf{0 , 2 8 3}$ & $\mathbf{0 , 1 8 7}$ & 0,8 & $\mathbf{0 , 7 1 1}$ \\
0,01 & 0,453 & 0,5 & 0,453 & $\mathbf{0 , 9 2 6}$ & 1,03 & $\mathbf{0 , 7 6 5}$ \\
$\mathbf{0 , 0 5}$ & 0,377 & 0,125 & 1,89 & $\mathbf{0 , 2 3 6}$ & 3,56 & $\mathbf{0 , 8 3 4}$ \\
$\mathbf{0 , 1}$ & 0,377 & 0,064 & 3,77 & $\mathbf{0 , 2 3 9}$ & 7,12 & $\mathbf{0 , 8 8 2}$ \\
0,5 & 0,377 & 0,013 & 18,85 & $\mathbf{0 , 2 4 5}$ & 35,54 & $\mathbf{0 , 4 1 5}$ \\
$\mathbf{1 , 0}$ & 0,377 & 0,0064 & 37,7 & $\mathbf{0 , 2 4 0}$ & 71,15 & $\mathbf{0 , 9 1 2}$
\end{tabular}

21. Entladungszeit 0,75 $\sigma . \quad w=19300+10000+100000=129300 \Omega$.

\begin{tabular}{l|l|l|l|l|l|l}
\hline 0,001 & 2,546 & 1,0 & 0,255 & $\mathbf{0 , 2 5 5}$ & 3,24 & $\mathbf{3 , 2 4}$ \\
0,005 & 1,146 & 0,667 & 0,573 & $\mathbf{0 , 3 6 2}$ & $\mathbf{3}, 29$ & $\mathbf{2 , 4 1 6}$ \\
0,01 & 1,0 & 0,4 & 1,0 & $\mathbf{0 , 4}$ & 5,0 & $\mathbf{3 , 2}$ \\
0,05 & 0,857 & 0,1 & 4,29 & $\mathbf{0 , 4 2 9}$ & 18,37 & $\mathbf{3}, \mathbf{4 9}$ \\
0,1 & 0,857 & 0,056 & 8,57 & $\mathbf{0 , 4 8}$ & $\mathbf{3 6 , 7 4}$ & $\mathbf{4 , 0 1 1}$ \\
$\mathbf{0 , 5}$ & 0,857 & 0,011 & 42,9 & $\mathbf{0 , 4 7 8}$ & 183,7 & $\mathbf{4 , 0 6}$ \\
1,0 & 0,889 & 0,00542 & 88,9 & $\mathbf{0 , 4 8 2}$ & 395,05 & $\mathbf{4 , 2 7}$
\end{tabular}

22. Entladungszeit 0,95 o. $w=18800+10000=28800 \Omega$.

\begin{tabular}{|c|c|c|c|c|c|c|}
\hline 0,001 & 2,203 & 1,0 & 0,22 & 0,22 & 2,425 & 2,425 \\
\hline 0,005 & 0.709 & 1,0 & 0,355 & $\mathbf{0 , 3 5 5}$ & 1,26 & 1,96 \\
\hline 0,01 & 0,47 & 1,0 & 0,474 & $0, \mathbf{1 7 4}$ & 1,11 & $\mathbf{1 , 1 0}$ \\
\hline 0,05 & 0,246 & 0,46 & 1,23 & 0,56 & 1,51 & 1,07 \\
\hline 0,1 & 0,23 & 0,25 & 2,3 & 0,575 & 2,65 & 1,16 \\
\hline 0,5 & 0,21 & 0,056 & 10,52 & 0,588 & 11,06 & 1,205 \\
\hline 1,0 & 0,21 & 0,028 & 21,03 & 0,588 & 22,11 & $\mathbf{1}, \mathbf{2 2}$ \\
\hline
\end{tabular}

23. ${ }^{\lambda}$ Entladungszeit 0,95 $\sigma . \quad w=21000+10000=31000 \Omega$

\begin{tabular}{l|l|l|l|l|l|l}
\hline $\mathbf{1 , 0}$ & 0,253 & 0,0266 & 25,3 & $\mathbf{0 , 6 7 3}$ & 32,08 & $\mathbf{1 , 7 1}$ \\
$\mathbf{0 , 5}$ & 0,253 & $\mathbf{0 , 0 5 3 4}$ & 12,65 & $\mathbf{0 , 6 7 6}$ & $\mathbf{1 6 , 0 4}$ & $\mathbf{1 , 6 7}$ \\
0,1 & 0,283 & 0,241 & 2,83 & $\mathbf{0 , 6 8 2}$ & $\mathbf{4 , 0 1}$ & $\mathbf{1 , 7 0}$ \\
0,05 & $\mathbf{0 , 3 5 7}$ & $\mathbf{0 , 4 6 7}$ & 1,79 & $\mathbf{0 , 8 3 2}$ & 3,19 & $\mathbf{9 , 2 3}$ \\
$\mathbf{0 , 0 1}$ & 0,651 & 0,857 & 0,65 & $\mathbf{0 , 5 5 8}$ & 2,12 & $\mathbf{9}, \mathbf{0 8}$ \\
$\mathbf{0 , 0 0 5}$ & 0,973 & $\mathbf{1}, 0$ & 0,487 & $\mathbf{0 , 4 8 7}$ & 2,37 & $\mathbf{2 , 3 7}$ \\
0,001 & 2,97 & $\mathbf{1 , 0}$ & 0,297 & $\mathbf{0 , 2 9 7}$ & 4,41 & $\mathbf{4 , 4 1}$
\end{tabular}

1) Dieser Versuch ist am gleichen Präparat nach Versuch 22 angestellt. 


\begin{tabular}{l|l|l|l|l|l|l|l|l}
\hline$c$ & $p$ & $\frac{r}{s}$ & $Q$ & $Q^{*}$ & $E$ & $E^{*}$ \\
\hline
\end{tabular}

24. Entladungszeit 1,15 $\sigma . \quad w=27300+10000=37300 \Omega$.

\begin{tabular}{l|l|l|l|l|r|l}
\hline 0,005 & 1,354 & 1,0 & 0,677 & $\mathbf{0 , 6 7 7}$ & 4,58 & $\mathbf{4 , 5 8}$ \\
0,01 & 0,919 & 0,9 & 0,919 & $\mathbf{0 , 8 2 7}$ & 4,22 & $\mathbf{4 , 1 8}$ \\
0,05 & 0,569 & 0,43 & 2,85 & $\mathbf{1 , 2 2 3}$ & 8,09 & $\mathbf{5 , 8 1}$ \\
0,1 & 0,521 & 0,26 & 5,21 & $\mathbf{1 , 0 5 5}$ & 13,59 & $\mathbf{6 , 0 2}$ \\
0,5 & 0,462 & 0,0586 & 23,1 & $\mathbf{1 , 3 5}$ & 53,25 & $\mathbf{6 , 0 5}$ \\
1,0 & 0,462 & 0,029 & $\mathbf{4 6 , 2}$ & $\mathbf{1 , 3 4}$ & 106,5 & $\mathbf{6 , 1 0}$
\end{tabular}

25. Entladungszeit 1,15 б. $w=126800+10000=136800 \Omega$.

Lange Nervenstrecke.

\begin{tabular}{l|l|l|l|l|l|l}
\hline $\mathbf{0 , 0 0 1}$ & 0,522 & 1,0 & 0,052 & $\mathbf{0 , 0 5 2}$ & 0,135 & $\mathbf{0 , 1 3 5}$ \\
0,005 & 0,144 & 0,875 & 0,072 & $\mathbf{0 , 0 6 3}$ & 0,052 & $\mathbf{0 , 0 5 1}$ \\
$\mathbf{0 , 0 1}$ & 0,0994 & 0,615 & 0,099 & $\mathbf{0 , 0 6 1}$ & 0,0498 & $\mathbf{0 , 0 4 2}$ \\
$\mathbf{0 , 0 5}$ & 0,0676 & 0,167 & 0,338 & $\mathbf{0 , 0 5 6}$ & 0,115 & $\mathbf{0 , 0 3 5}$ \\
$\mathbf{0 , 1}$ & 0,0629 & 0,087 & 0,629 & $\mathbf{0 , 0 5 5}$ & 0,198 & $\mathbf{0 , 0 3 3}$ \\
0,5 & 0,0629 & 0,017 & 3,145 & $\mathbf{0 , 0 5 3}$ & 0,991 & $\mathbf{0 , 0 3 4}$ \\
$\mathbf{1 , 0}$ & 0,0629 & 0,0087 & 6,29 & $\mathbf{0 , 0 5 5}$ & 1,982 & $\mathbf{0 , 0 3 4}$
\end{tabular}

26. Entladungszeit 1,15 $\sigma . \quad w=22800+10000+100000=132800 \Omega$.

\begin{tabular}{l|l|l|l|l|l|l}
\hline 0,001 & 1,394 & 1,0 & 0,139 & $\mathbf{0 , 1 3 9}$ & 0,972 & $\mathbf{0 , 9 7 2}$ \\
0,005 & 0,569 & 0,83 & 0,285 & $\mathbf{0 , 2 3 7}$ & 0,809 & $\mathbf{0 , 5 8 7}$ \\
0,01 & 0,491 & 0,6 & 0,491 & $\mathbf{0 , 2 9 5}$ & 1,203 & $\mathbf{1 , 0 1 1}$ \\
0,05 & 0,394 & 0,146 & 1,97 & $\mathbf{0 , 2 8 8}$ & 3,873 & $\mathbf{1 , 0 5 1}$ \\
$0, \mathbf{1}$ & 0,394 & 0,075 & 3,94 & $\mathbf{0 , 2 9 6}$ & 7,75 & $\mathbf{1 , 1 0 7}$ \\
0,5 & 0,397 & 0,014 & 19,85 & $\mathbf{0 , 2 7 8}$ & 39,37 & $\mathbf{1 , 1 4 3}$ \\
1,0 & 0,403 & 0,007 & 40,3 & $\mathbf{0 , 2 8 2}$ & 81,3 & $\mathbf{1 , 1 5 8}$
\end{tabular}

$27^{1}$ ). Entladungszeit $2 \sigma . \quad w=38400+10000+100000=148400 \Omega$.

\begin{tabular}{l|l|l|l|l|l|l}
\hline $\mathbf{1}, 0$ & 0,476 & 0,0114 & 47,58 & $\mathbf{0 , 5 4 2}$ & 113,2 & $\mathbf{2 , 5 6 7}$ \\
0,001 & 1,846 & 1,0 & 0,185 & $\mathbf{0 , 1 8 5}$ & 1,705 & $\mathbf{1 , 7 0 5}$ \\
0,001 & 1,694 & 1,0 & 0,169 & $\mathbf{0 , 1 6 9}$ & 1,436 & $\mathbf{1 , 4 3 6}$ \\
$\mathbf{1 , 0}$ & 0,421 & 0,0118 & 42,06 & $\mathbf{0 , 4 9 6}$ & 88,45 & $\mathbf{2 , 0 7 5}$
\end{tabular}

28. Entladungszeit $2 \sigma . \quad w=41800+10000+100000=151800 \Omega$.

\begin{tabular}{l|l|l|l|l|l|l}
\hline $\mathbf{1}, 0$ & 0,364 & 0,0125 & 36,36 & $\mathbf{0 , 4 5 5}$ & $66, \mathbf{1}$ & $\mathbf{1 , 6 4 2}$ \\
0,001 & 1,496 & 1,0 & 0,15 & $\mathbf{0 , 1 5}$ & 1,119 & $\mathbf{1 , 1 1 9}$ \\
$\mathbf{0 , 0 0 1}$ & $\mathbf{1 , 4 3 6}$ & 1,0 & 0,144 & $\mathbf{0 , 1 4 4}$ & 1,03 & $\mathbf{1 , 0 3}$ \\
$\mathbf{1 , 0}$ & $\mathbf{0 , 3 5 5}$ & 0,0103 & 35,53 & $\mathbf{0 , 3 6 6}$ & 63,15 & $\mathbf{1 , 2 9 4}$
\end{tabular}

1) Versuch 27 und 28 sind mit Unterbrechung durch Versuch 31 an demselben Präparat angestellt. 


$=$

29. Entladungszeit $3 \sigma . w=48900+10000+100000=158900 \Omega$.

Entladungsstrom absteigend.

\begin{tabular}{|c|c|c|c|c|c|c|}
\hline 1,0 & 0,377 & 0,017 & 37,73 & 0,641 & 71,15 & $\mathbf{2 , 3 9 3}$ \\
\hline 0,5 & 0,4 & 0,034 & 20,0 & 0,680 & 40,0 & $\overrightarrow{\mathbf{2}, 654}$ \\
\hline 0,1 & 0,485 & 0,145 & 4,35 & 0,631 & 9,44 & 2,530 \\
\hline 0,05 & 0,476 & 0,288 & 2,38 & 0,686 & 5,67 & 2,7966 \\
\hline 0,01 & 0,649 & 0,86 & 0,65 & 0,559 & 2,11 & $\overline{\mathbf{2}}, 059$ \\
\hline 0,005 & 0,836 & 1,0 & 0,417 & 0,417 & 1,745 & $\mathbf{1}, 74 \overline{3}$ \\
\hline 0,001 & 1,921 & 1,0 & 0,192 & 0,192 & 1,845 & 1,845 \\
\hline
\end{tabular}

Entladungsstrom aufsteigend.

\begin{tabular}{l|l|l|l|l|l|l}
\hline 1,0 & 0,393 & 0,0193 & 33,33 & $\mathbf{0 , 6 4 3}$ & 55,55 & $\mathbf{2 , 1 2 4}$ \\
0,5 & 0,367 & 0,032 & $\mathbf{1 8 , 3 6}$ & $\mathbf{0 , 5 8 8}$ & 33,74 & $\mathbf{2 , 1 2 5}$ \\
0,1 & 0,4 & 0,163 & 4,0 & $\mathbf{0 , 6 5 2}$ & 8,0 & $\mathbf{2 , 3 9 5}$ \\
0,05 & 0,459 & 0,28 & 2,29 & $\mathbf{0 , 6 4 1}$ & 5,26 & $\mathbf{2 , 5 0 3}$ \\
0,01 & 0,646 & 0,786 & 0,65 & $\mathbf{0 , 5 1 1}$ & 2,095 & $\mathbf{2 , 0 3 5}$ \\
0,005 & 0,835 & 1,0 & 0,417 & $\mathbf{0 , 4 1 7}$ & 1,745 & $\mathbf{1 , 7 4 5}$ \\
0,001 & 1,886 & 1,0 & 0,189 & $\mathbf{0 , 1 8 9}$ & 1,778 & $\mathbf{1 , 7 7 8}$
\end{tabular}

Die Versuche dieser Reihe wurden zeitlich mit der Stromrichtung alternierend angestellt und sind nur zur besseren Übersicht getrennt verzeichnet.

30. Entladungszeit อั $\sigma . \quad w=12000+10000+100000=122000 \Omega$.

\begin{tabular}{|c|c|c|c|c|c|c|}
\hline 1,0 & 1,111 & $0,0.546$ & 111,1 & $\mathbf{3}, \mathbf{4 4 2}$ & 616 & 41,86 \\
\hline 0,5 & 1,273 & 0,0705 & 61,37 & 4,324 & 404,95 & 55,08 \\
\hline 0,1 & 1,441 & 0,293 & 14,41 & 4,222 & 103,8 & 51,91 \\
\hline 0,05 & 1,441 & 0,507 & 7,21 & $\mathbf{3}, 552$ & 51,9 & 39,29 \\
\hline 0,01 & 2,222 & 0,92 & 2,22 & $\mathbf{2 , 0 4 2}$ & 24,69 & 24,53 \\
\hline 0,005 & 2,491 & 0,99 & 1,25 & 1,283 & 15,5 & $\mathbf{1 5 , 5}$ \\
\hline
\end{tabular}

31. Entladungszeit 5 $\sigma, \quad w=40600+10000+100000=150600 \Omega$.

\begin{tabular}{l|l|l|r|r|r|r}
\hline $\mathbf{1}, 0$ & 0,3936 & 0,028 & 39,36 & $\mathbf{1 , 1 0 1}$ & 77,45 & $\mathbf{4 , 2 7 7}$ \\
0,5 & 0,4064 & 0,054 & 20,32 & $\mathbf{1 , 0 9 7}$ & 41,28 & $\mathbf{4 , 3 3 8}$ \\
0,1 & 0,4064 & 0,25 & 4,06 & $\mathbf{1 , 0 1 6}$ & 8,26 & $\mathbf{3 , 6 1 4}$ \\
0,05 & 04704 & 0,44 & 2,35 & $\mathbf{1 , 0 3 5}$ & 5,54 & $\mathbf{3 , 7 9 9}$ \\
0,01 & 0,6418 & 0,99 & 0,64 & $\mathbf{0 , 5 9 7}$ & 2,06 & $\mathbf{2 , 0 5 1}$ \\
0,005 & 0,797 & 1,0 & 0,399 & $\mathbf{0 , 3 9 9}$ & $\mathbf{1 , 5 9}$ & $\mathbf{1 , 5 9 0}$ \\
0,001 & 1,773 & 1,0 & 0,177 & $\mathbf{0 , 1 7 7}$ & $\mathbf{1 , 5 7}$ & $\mathbf{1 , 5 7 1}$
\end{tabular}

\section{Folgerungen aus den Versnchen und Theoretisches.}

1. Der Quantitäts- und Energiebedarf.

a) Die Versuche mit kürzesten Entladungszeiten $(0,1$ und 0,2 o).

Folgende einfache Überlegung zeigt, dass zur Feststellung der wirklichen zur Reizung erforderlichen Elektrizitäts- und Energiemengen hauptsächlich die Versuchsreihen mit $\mathrm{k} u ̈ \mathrm{rz}$ este $\mathrm{r}$ Ent- 
ladungszeit geeignet sein werden. Die Versuche nach dem Verfahren von Lapi cque (vgl. oben S. 174 und 183 f.) haben ergeben, dass ein um so kleinerer Teil der vorhandenen Quantität wirklich zur Verwendung kommt, je grösser die Kapazität. Bei der kleinsten Kapazität ging aber die Nutzzeit niemals unter $0,1 \sigma$ herab. Bei $0,1 \sigma$ ist man also für alle verwendeten Kapazitäten sicher, dass kein Teil der sich entladenden Quantität oder Energie unausgenützt bleibt.

Die Versuchsreihen mit Entladungszeiten von 0,1 und für einen grossen Kapazitätsbereich auch die mit $0,2 \sigma$ oder etwas mehr müssen nach dem Gesagten unser hauptsächliches Interesse beanspruchen. Deswegen haben wir auch besonders zahlreiche Versuche mit $0,1 \sigma$ Entladungszeit angestellt.

Zunächst sei folgendes bemerkt: Bej vollständiger Entladung zeigen bekanntlich die erforderlichen Energien sehr regelmässig bei einer gewissen Kapazität ein Minimum. 'Die hi er angeführten $E$-Beträge, d. h. diejenigen Energien, welche der Kondensator enthalten muss, um bei der zugelassenen Partialentladung die Minimalzuckung zu geben, zeigen dieses Verhalten nicht, während es sich S. 184 zeigte, weil im Lapicque-Versuch die $E$ dieselbe Bedeutung haben, wie in den älteren Versuchen.

Was dem Leser in den Versuchen mit kürzesten Entladungszeiten sofort auffallen wird, ist die sehr geringe Variation der $Q^{*}$ und oft auch der $E^{*}$-Werte im Laufe einer die Kapazitäten von 1 bis 0,001 Mf. umfassenden Versuchsreihe; für die Quantitäten drängte sich dies schon beim Versuche selbst durch die auffallende Konstanz der $r$-Ablenkingen auf. Die nirgends ganz fehlenden Abweichungeu von der Konstanz liegen anscheinend innerhalb der sicher sehr erheblichen Fehlerbreiten. Von Abweichungen tritt namentlich häufig eine Zunahme des Bedarfs im Laufe des Versuchs hervor, und zwar regelmässiger und stärker beim Energie- als beim Quantitätsbedarf. Diese Zunahme ist sowohl in Reihen mit steigenden wie in solchen mit fallenden Kapazitäten bemerklich, und auch in Versuch 6 , in welchem absichtlich im Interesse dieser Frage eine regellose Folge der Kapazitäten gewählt wurde. Es unterliegt wohl kaum einem Zweifel, dass die Ursache dieser zeitlichen Bedarfszunahme in der Ermüdung des Präparats zu suchen ist, welche wegen der hohen erforderlichen Potentiale (oft über 3 , zuweilen fast 5 Volt) nicht gering sein wird. Dass von den hier abgeleiteten Regeln auch Aus- 
nahmen vorkommen, muss ausdrücklich erwähnt werden. Es darf aber vermutet werden, dass bei ganz ausgeschlossener Ermüdung in diesen Versuchen, deren Charakteristikum ist, dass jeder unausgenützte Elektrizitätsaufwand vermieden wird, sich ein von der Kapazität unabhängiger Quantitäts- od e $r$ Energiebedarf berausstellen würde.

Die Versuche reichen unseres Erachtens, trotzdem sie mit aller erdenklichen Sorgfalt angestellt sind, nicht aus, um sicher zu entscheiden, ob die Quantitäts- oder die Energiebeträge von den Kapazitäten unabhängiger sind, oder ob - auch diese Möglichkeit muss zugegeben werden - die Konstanz für keine der beiden Grössen zutrifft.

Auf die grossen Fehlerbreiten aller Versuche dieses Gebiets ist hier schon wiederholt hingewiesen worden. Wenn es infolgedessen schon unwahrscheinlich ist, dass die Versuche die unbekannte wahre Gesetzmässigkeit obne weiteres enthüllen werden, so ist ausserdem ein leicht übersehbares, durch eine einfache Formel ausdrückbares Verhalten gar nicht zu erwarten, da zwischen dem an der Reizstelle des Nerven einwirkenden elektrischen Vorgang einerseits und den mechanischen und chemischen Vorgängen im Muskelprotoplasma, welche die Kontraktion unmittelbar herbeiführen, andrerseits eine grosse Reihe von Zwischengliedern existiert, deren gegenseitige Beziehungen schwerlich einfach linearer Natur sind. Also mehr als rohe Annäherung wird kaum zu erreichen sein.

Eine solche Annäherung ist es nun, dass bei Vermeidung jedes unausgenützten Anteils elektrischer Einwirkung eine von der Kapazität unabhängige Zeitsumme, sei es von Quantität, sei es von Energie für die Minimalzuckung erforderlich ist.

Selbstverständlich kann nicht davon die Rede sein, dass etwa sow o h $1 Q^{*}$ als $E^{*}$ konstant sein könnte. Setzt man nämlich in den Gleichungen (1) b e i d e Grössen konstant, so ergibt sich durch gegenseitige Division, dass dann auch die Gröse $p\left(1+e^{-\frac{t}{w c}}\right)$ konstant sein müsste, was im böchsten Grade unwahrscheinlich ist.

Weiter aber lässt sich leicht zeigen, dass, wenn $Q^{*}$ konstant ist, in der Tat auch die $E^{*}$-Werte nur wenig von der Konstanz abweichen können. Aus (1) folgt nämlich

$$
e^{-\frac{t}{t c c}}=1-\frac{Q^{*}}{p c}
$$


Setzt man dies in den Ausdruck für $E^{*}$ ein, so ergibt sich

$$
E^{*}=\frac{1}{2} p^{2} c\left[1-\left(1-\frac{Q^{*}}{p c}\right)^{2}\right] \quad \text { oder } \quad E^{*}=Q^{*}\left(p-\frac{Q^{*}}{2 c}\right)
$$

d. h. wenn $Q^{*}$ konstant (z. B. $=2 b$ ) ist, so würde auch $E^{*}$ einen konstanten Betrag aufweisen, wenn $p-\frac{b}{c}$ ebenfalls konstant, z. B. $=a$ wäre, oder wenn $p=a+\frac{b}{c}$ wäre. Dies ist aber die Hoorw eg'sche Gleichung für $p$, welche, wenn auch, wie ich gezeigt habe, durchaus nicht genau, so doch mit einer gewissen Annäherung gilt ${ }^{1}$ ). Man. kann also sagen: einem wirklich konstanten $Q^{*}$ entspricht $\mathrm{mit}$ einer gewissen Annäherung auch ein konstantes $E^{* 2}$ ).

Diese Betrachtung lässt sich noch weiter fortführen. Meine Versuche baben auf das bestimmteste ergeben, dass die eben erwähnte Hoorweg'sche Gleichung zwar für einen gewissen Bereich von Kapazitäten eine genügende Annäherung bietet, aber eine absolut regelmässige systematische Abweichung existiert, insofern nach den höheren Kapazitäten die wirklichen $p$-Werte immer mehr hinter den berechneten zurückbleiben $\left.{ }^{3}\right)$, d. h. der Betrag $p-Q^{*} / 2 c$ ist, wenn $Q^{*}$ konstant ist, zwar für eine gewisse Strecke leidlich konstant, nimmt aber nach den grösseren Kapazitäten systematisch $a b$. Hiernach ist zu erwarten, dass bei konstantem Quantitätsbedarf eine Zunahme des Energiebedarfs mit abnehmender Kapazität stattfindet. Eine genauere Prüfung der Versuchszahlen ergibt, dass zwar vielfach in der Tat die $E^{*}$-Beträge mit abnehmender Kapazität steigen, während gleichzeitig die $Q^{*}$ ziemlich unverändert bleiben, dass aber auch das Entgegengesetzte vorkommt, und das erstere meist (s. oben) in Wirklichkeit auf Ermüdung zurückzuführen ist.

Man kann sich nun weiter fragen, ob eine massgebende Bedeutung der Quantität oder der Energie wahrscheinlicher ist. Der Vorgang im Muskel ist so unverständlich, dass man sich bis auf weiteres ebensogut damit abfinden könnte, dass der Muskel die

1) In meiner letzten Arbeit (dieses Arch. Bd. 111 S. 542-546) ist für alle Versuche der wahrscheinlichste Wert von $b$ berechnet. Setzt man $Q^{*}=2 b$, so erhält man überall einen Betrag, welcher der Quantität für das Optimum ziemlich nahe kommt.

2) Mit den gemachten Annahmen würde $E^{*}=2 a b$ werden.

3) Vgl. dieses Archiv Bd. 111 S. 542_546. Dieselbe Erscheinung zeigen auch die Versuche anderer Autoren (vgl. ebendaselbst.S. 540). Die Vorzeichen der dort angegebenen Abweichungen bedeuten berechnete minus gefundene Werte. 
differentiellen Einwirkungen $i d t$, wie dass er die $w i^{2} d t$ zeitlich summiert. Aber folgende Erwägungen machen die Berleutung der Energie wahrscheinlicher. Erstens müsste, wenn es auf die Quantitäten ankäme, dies auch in den Versuchen nach Lapicque's Verfahren $\mathrm{zu}$ erkennen sein, da auch in diesen eine vollständige Ausnützung des elektrischen Aufwandes stattfindet; bier sieht man aber sowohl in Lapieque's Beispiel als auch in Herrn Adam's Versuchen die $Q^{*}$-Beträge keineswegs konstant, sondern mit der Kapazität wacbsend. In diesen Versuchen zeigt sich vielmehr eine ziemliche Konstanz der Energi e beträge, wenigstens insofern, als dieselben bei der kleinsten und der grössten Kapazität nur wenig verschieden sind, dazwischen allerdings ein Minimum durchlaufen.

Ferner spricht der Umstand, dass bei Vollentladungen so ungemein regelmässig die Energie, keineswegs aber die Quantität, hei einer gewissen Kapazität ein Minimum durchläuft, ausserordentlich dafür, dass die Energie eine massgebende Rolle spielt. Wie ich schon in meiner letzten Arbeit entwickelt habe, ist die natürlichste Deutung die, dass bei dieser Kapazität der elektrische Vorgang am günstigsten ausgenützt wird. Meine hierfür versuchte Erklärung, dass bei der optimalen Kapazität der wesentliche Teil der Entladung grade die Latenzzeit ansfüllt, mag irrig sein; etwas anderes an ihre Stelle zu setzen, sehe ich vorläufig keinen Weg. Sehr bemerkenswert erscheint es aber, dass (S. 184) die Beträge $E^{*}$ bei ungefähr denselben Kapazitäten wie die $E$-Beträge ein Minimum aufweisen, mit anderen Worten also diese Kapazität auch dann optimal ist, wenn jeder unausgenützte Elektrizitätsaufwand vermieden ist. Dies scheint mir von neuem für den Gerlanken zu sprechen, dass die dieser optimalen Kapazität entsprechende Nutzzeit, d. h. ungefähr Zeiten zwischen 0,3 und $0,9 \sigma$ für den Muskel eine Art kritische Bedeutung haben, dergestalt, dass eine sich über diese Zeit erstreckende Einwirkung mit dem geringsten Energieaufwand die Zuckung auslöst.

Ein weiterer Umstand, welcher unsere Aufmerksamkeit mehr auf die Energie als auf die Quantität als den massgebenden Faktor zu lenken geeignet ist, besteht darin, dass Nernst (siehe den letzten Teil dieser Arbeit) die Frgebnisse der Versuche mit kurzen Schliessungen konstanter Ströme und mit Wechselströmen durch eine Formel darstellen zu können behauptet, zu welcher er auf theoretischem Wege gelangt ist, und welche darauf hinauslaufen 
würde, dass der Energiebedarf von den Versuchsvariablen unabhängig ist, und dass Eucken auf gleichem Wege auch für Kondensatorreizung zu einer analogen Formel gelangt.

\section{b) Die Versuche mit Entladungszeiten zon 0,3 bis 5 .}

Für etwas längere, aber noch immer unter etwa $3-5 \sigma$ liegende Entladungszeiten ist folgendes $\mathrm{zu}$ erwägen. Ist $t$ die Entladungszeit und $u$ die Nutzzeit, so wird, wo $t<u$ ist, kein Teil der zugelassenen Entladung unausgenützt bleiben; wo dagegen $t>u$ ist, wird mehr ausgegeben als ausgenützt. Im ersteren Falle muss sich also der Quantitäts- und Energiebedarf so verhalten, wie in den Versuchen mit $t=0,1 \sigma$, in welchen durchweg $t<u$ war. Dieser Fall findet in unseren Versuchen durcbgehends statt bei den grössten Kapazitäten, und reicht in die kleineren um so weiter herab, je kleiner $t$. Dagegen ist $t>u$ besonders bei den kleineren Kapazitäten, und dies reicht in die höheren um so weiter hinauf, je grösser $t$.

Hiernach ist auf Grund unserer bisherigen Ergebnisse zu erwarten, dass bei allen verwendeten Entladungszeiten für die höheren Kapazitäten der sich herausstellende Quantitäts- resp. Energiebedarf mit abnehmender Kapazität sich kaum ändert, bei weiterer Abnahme der Kapazität aber entschieden immer kleiner wird. Der Bereich konstanten Bedarfs muss aber um so weiter zu kleineren. Kapazitäten herabreichen, je kürzer die Entladungszeit.

Dies Verhalten zeigt sich nun wirklich in den Versuchen so weit, wie es bei den unvermeidlichen Fehlerquellen irgend $\mathrm{zu}$ erwarten ist. Bei $t=5 \sigma$ (Versuch 30 und 31) erstreckt sich der Bereich annähernd konstanten Bedarfs von 1,0 bis 0,1 oder 0,05 Mf.; ähnlich ist es bei $t=3 \sigma$ (Versuch 29). Unterhalb der Konstanzgrenze sieht man den Bedarf entsebieden mit der Kapazität sinken. Bei $0,5 \sigma$ (Versuch 17) ist die Konstanzgrenze schon bis 0,005 Mf. herabgerückt. Dass das Herabrücken der Grenze nicht mit vollkommener Regelmässigkeit verläuft, wird niemand überraschen, zumal wir oben S. 185 gesehen haben, wie sehr die $u$-Werte, auf die es hier ankommt, bei verschiedenen Präparaten variieren. Es bedarf kaum der Erwähnung, dass, wenn man $t$ immer grösser (über $5 \sigma$ ) wachsen liesse, das Verhalten sich immer mehr demjenigen bei vollständiger Entladung nähern, d. h. eine starke Abnahme des Quantitätsbedarfs mit der Kapazität stattfinden würde. 


\section{Zusammenfassung und allgemeinere Schlüsse.}

Die Versuche haben ungefähr folgendes ergeben:

1. Bei vollständiger Ausnützuug der entladenen Elektrizität ist zur Minimalzuckung bei jeder Kapazität annähernd dieselbe Energiesumme erforderlich.

2. Wird bei vollständiger Ausnützung die Entladungszeit entsprechend der Kapazität variiert (L a picque-Versuche), so zeigt sich ein Optimum insofern, als bei einer gewissen mittleren Kapazität (und Entladungszeit) etwas weniger Energie nötig ist als sonst.

Die vorstehenden Ergebnisse sind rein empirisch gewonnen, ohne dass eine Theorie oder Hypothese über die Natur der indirekten elektrischen Muskelreizung uns vorgeschwebt hätte; auch habe ich selbst nie eine solche aufzustellen versucht, abgesehen von der wiederholt gemachten Bemerkung, dass der Muskel, abweichend vom Nerven, Differentialerregungen zeitlich summiert, worauf übrigens alle Erfahrungen dieses Gebietes nachdrücklich hinweisen.

Ob diese Summation schon im Nerven oder erst im Muskel stattfindet, kann vorläufig nicht endgültig entschieden werden; aber mindestens ist das letztere bei weitem wahrscheinlicher. Nach meiner Theorie der Erregungsleitung und den sich an dieselbe anschliessenden Versuchen ${ }^{1}$ ) wird jeder auf eine Nervenstelle einwirkende elektrische Vorgang wellenartig durch die Kontinuität der Faser fortgeleitet und reproduziert sich überall mit Erhaltung seines zeitlichen Verlaufs ${ }^{2}$ ), etwa wie eine lokale Einwirkung auf ein vollkommen elastisches Medium, nur mit denjenigen Modifikationen, wie sie einer Art von Dämpfung, analog der Reibung beim elastischen Medium, entsprechen ${ }^{3}$ ). Wenn also ein Kondensator sich durch

1) Dieses Archiv Bd. 75 S. 574. 1899; Ann. d. Physik. Vierte Folge Bd. 12 S. 932. 1908, und (mit Gildemeister) Bd. 14 S. 1031. 1904; ferner dieses Archiv Bd. 109 S. 95. 1905.

2) Bei Schliessung eines konstanten Stroms entsteht ebenfalls ein wellenartig ablaufender Vorgang, welcher in den stationären Elektrotonus abklingt (s. hierüber Bd. 109 S. 124).

3) Die jetzt wesentlich vervollkommneten Mittel, das Aktionspotential im Nerven direkt zu verfolgen (Saitengalvanometer usw.), sind bisher noch nicht benutzt worden, dieses theoretische Ergebnis zu prüfen, und es ist nicht sicher, ob sie dazu ansreichen werden. 
einè Nervenstrecke entlädt, so findet nach Ablauf der Leitungszeit ein analoger Vorgang am intramuskulären Nervenende statt, und es ist nicht viel anders, als wenn die Entladung auf den Muskel direkt gewirkt hätte ${ }^{1}$ ).

$\mathrm{Ob}$ nun die Auslösung der Zuckung, welche offenbar erst durch eine Summe differentieller elektrischer Effekte zustande kommt, eine Quantitäts- oder eine Energiewirkung ist, ob also die Elemente $i d t$ oder $w i^{2} d t$ summiert werden, muss als noch nicht endgültig entschieden bezeichnet werden, obwohl das letztere wahrscheinlicher ist.

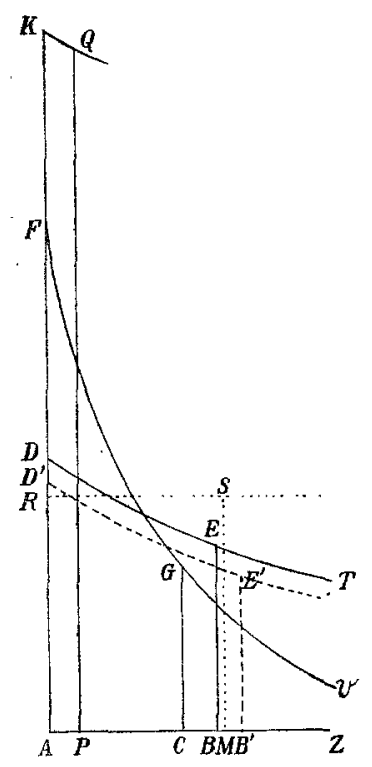

Fig. 2.

Von der Entscheidung dieser Frage wird die zukünftige Theorie der elektrischen Muskelreizung abhängen, und diese wird auch über das Wesen der Latenzzeit sowie über Lapicque's Nutzzeit Aufschluss geben müssen. In Fig. 2 habe ich schematisch die Verhältnisse dargestellt, welche sich aus dem Versuch von Lapicque ergeben, und zwar für zwei verschiedene Kapazitäten. Die Abszissen sind Zeiten, die Ordinaten Energien. Die Kurve $A D E B$ stellt den Energieverlauf für 1 Mf. dar, $A B$ die Nutzzeit, $A D$ die zur Zuckung nötige Anfangsenergie ${ }^{2}$ ). Die Versuche lehren, dass erstens der Teil $T E B Z$ der Entladung zur Erregung nichts beiträgt, zweitens der Vorgang $A D E B$ nicht durch den Vorgang $A D^{\prime} E^{\prime} B^{\prime}$ mit etwas niedrigerer Anfangsintensität etwa dadurch ersetzt werden kann, dass die Entladung etwas länger ausgenützt wird, so dass die Fläche $A D E^{\prime} B^{\prime}$ gleiche Energie repräsentieren würde wie $A D E B$. Auf keinen Fall also stellt ein Energiequantum für sich die Bedingung der Minimalzuckung dar, ebensowenig eine Anfangsstromstärke oder

1) Einige Versuche über direkte Kondensatorreizung des Muskels siehe im nächsten Abschnitt.

2) Bemerkt sei noch, dass die Kurven ebenso aussehen würden, wenn die Ordinaten durchweg statt der Energien Stromstärken, die Flächen also statt Energiesummen Elektrizitätsmengen darstellten. Nur würde dann jede Abszisse eine doppelt so lange Zeit bedeuten, als wenn Energien gemeint sind. 
Anfangsenergie für sich. Die durch $A B$ dargestellte Nutzzeit für 1 Mf. ist von derselben Grössenordnung wie die in meiner vorigen Arbeit als kritische Zeit oder annähernde Latenzzeit des Muskelelements hingestellte, und man könnte noch jetzt annehmen, dass der unwirksame Teil $T E B Z$ der Entladung nur deswegen nicht wirkt, weil zur Zeit $B$ im Muskel die die Zuckung vorbereitenden Prozesse schon beendet sind. Dann würde also $A D$ derjenige Anfangsbetrag sein, der innerhalb der allein in Betracht kommenden Zeit $A B$ bei 1 Mf. grade die der geforderten Energiesumme entsprechende Fläche $A D E B$ ergibt.

Die Kurve $F U$ stelle nun den Energieabfall für eine kleinere Kapazität, z. B. 0,01 Mf., dar; die ihr entsprechende Nutzzeit sei $A C$ und $A F G C$ die jetzt erforderliche Energiefläche, $A F$ die zu ihrer Herstellung in der Nutzzeit erforderliche grössere Anfangsenergie. Wiederum ist der Teil $U G C Z$ der Entladung vollkommen wirkungslos, und der Vorgang. $A F G C$ lässt sich nicht durch einen etwas niedrigeren, aber dafür etwas längeren ersetzen. Nach den Versuchen (siehe obeu S. 174, 184) ist die Fläche $A F G C$ etwas kleiner als $A D E B$, vor allem aber die Nutzzeit $A C$ kürzer als $A B$. Wirklich erklären lässt sich dies, soviel ich sehe, vorläufig nicht; man wird sich mit der schon S. 173 angedeuteten Annahme begnügen müssen, dass mit der höheren Anfangswirkung eine Verkürzung der Vorbereitungszeit verbunden ist,

Die Kurve $A K Q P$ der Fig. 2 stellt noch den Fall einer a bgebrochenen Entladung für $1 \mathrm{Mf}$. dar; die Entladungszeit $A P$ möge z. B. $0,1 \sigma$ bedeuten. Es ist vorausgesetzt, dass die Fläche $A K Q P$ annähernd $=A D E B$ sein muss.

Zwischen dem Verhalten bei hoher Kapazität (z. B. 1 Mf.) und demjenigen bei unendlicher Kapazität, d. h. konstantem Strom, kann kein prinzipieller Unterschied, sondern nur ein kontinuierlicher Übergang sein. So habe ich in meiner vorigen Arbeit fur beide Fälle dasselbe Grenzpotential gefunden (Bd. 111, S. 547). Daher ist für Schliessung eines konstanten Stromes ebenfalls eine Nutzzeit zu erwarten, welche höchstens eine Spur länger sein wird als bei 1 Mf. Die punktierte Kurve $A R S M$ stellt gemäss dieser Anschauung den wirksamen Teil der Schliessungszeit, VSMZ den wirkungslosen Rest dar. Leider war es uns aus einem experimentellen Grunde bisher nicht möglich, den Energiebedarf bei Zeit- 
schliessung und bei Kondensatorreizung mit 1 Mf. unmittelbar zu vergleichen ${ }^{1}$ ); jedoch beabsichtigen wir diese Lücke auszufüllen.

Einige Versuche von Lapicque ${ }^{2}$ ) befassen sich mit dieser Frage. $\mathrm{Er}$ bestimmte für eine gegebene Kapazität das erforderliche Potential und die Nutzzeit und hierauf das Potential, das für eine Zeitschliessung von der Dauer der letzteren erforderlich war. Von den Galvanometerablenkungen, welche diese beiden Reizströme gaben, war die erstere etwas kleiner als die letztere, woraus er schliesst, 'dass die Kondensatorreizung kleinere Quantität braucht als die Zeitschliessung. In der folgenden kleinen Tabelle, welche diese Versuche wiedergibt ${ }^{3}$ ), habe ich in den beiden letzten Rubriken die nach den Formeln (1) berechneten Quantitäts- und Energiewerte hinzugefügt, die allerdings den Einwand zulassen, dass bei Lapicque der Einfluss der Selbstinduktion usw. weniger zu vernachlässigen war als bei uns. In drei von den vier Versuchspaaren bestätigt sich auch so

\begin{tabular}{|c|c|c|c|c|c|c|}
\hline \multicolumn{5}{|c|}{ Lapicque's Zahlen } & \multicolumn{2}{|c|}{ Von mir berechnet } \\
\hline$\stackrel{w}{\mathrm{Ohm}}$ & $\begin{array}{c}c \\
\text { MIf. }\end{array}$ & $t$ & $\begin{array}{c}p \\
\text { Volt }\end{array}$ & $r$ & $\begin{array}{c}Q^{*} \\
\text { (Verhältni }\end{array}$ & $\begin{array}{c}E^{*} \\
\text { zahlen) }\end{array}$ \\
\hline $\begin{array}{l}28500 \\
28500\end{array}$ & $\begin{array}{l}0,05 \\
\infty\end{array}$ & $\begin{array}{l}1,22 \\
1,22\end{array}$ & $\begin{array}{l}6,10 \\
4,10\end{array}$ & $\begin{array}{l}52 \\
54\end{array}$ & $\begin{array}{l}1754 \\
1755\end{array}$ & $\begin{array}{l}7625 \\
7196\end{array}$ \\
\hline $\begin{array}{l}28500 \\
28500\end{array}$ & $\begin{array}{l}0,02 \\
\infty\end{array}$ & $\begin{array}{l}0,67 \\
0,67\end{array}$ & $\begin{array}{l}8,75 \\
5,35\end{array}$ & $\begin{array}{l}30 \\
36\end{array}$ & $\begin{array}{l}1210 \\
1255\end{array}$ & $\begin{array}{l}6925 \\
6729\end{array}$ \\
\hline $\begin{array}{l}27000 \\
27000\end{array}$ & $\begin{array}{l}0,05 \\
\infty\end{array}$ & $\begin{array}{l}1,40 \\
1,40\end{array}$ & $\begin{array}{l}1,25 \\
0,82\end{array}$ & $\begin{array}{l}13 \\
16\end{array}$ & $\begin{array}{l}403,4 \\
425,2\end{array}$ & $\begin{array}{l}341,5 \\
348,6\end{array}$ \\
\hline $\begin{array}{l}27000 \\
27000\end{array}$ & $\begin{array}{c}0,02 \\
\infty\end{array}$ & $\begin{array}{l}0,50 \\
0,50\end{array}$ & $\begin{array}{l}1,95 \\
1,25\end{array}$ & $\frac{6-7}{9}$ & $\begin{array}{l}235,5 \\
231,5\end{array}$ & $\begin{array}{l}320,5 \\
289,4\end{array}$ \\
\hline
\end{tabular}

1) Solche Versuche würden voraussetzen, dass die erste Öffinang (bei $O_{1}$, Fig. 1) durch eine genan in dieselbe Zeit fallende Schliessung des konstanten Stromes ersetzt wird, der bei $O_{2}$ zu öffnen wäre. Nun besitzt zwar unser Institut vorzügliche Schliesskontakte für das Pendel, trotzdem war ihre Vertauschung mit dem Öffnungskontakt bei genauer Erhaltung des Moments bisher nicht ausführbar. An Stelle der Schliessung die Öffnung einer Nebenschliessung zum Nerven zu setzen, liegt zwar sehr nahe, scheitert aber daran, dass ein Punktkontakt niemals einen Strom vom Nerven ganz abblenden kann. Wir werden aber das Ziel durch andere Massnahmen zu erreichen wissen. - Das Schussrheotom van G. Weiss war für diese Aufgabe ohne weiteres verwendbar.

2) Compt. rend. de la soc. de biol. 1907 t. 1 p. 703; Journ. de physiol. et de pathol. 1907 p. $574 \mathrm{f}$.

3) Die Buchstaben im Kopfe haben dieselbe Bedeutung wie in den Versuchen unserer Arbeit. $e=\infty$ bedeutet, dass ein konstanter Strom verwendet ist. 
der geringere Quantitätsbedarf des Kondensatorversuchs; der Energiebedarf ist aber umgekehrt in drei von den vier Versuchspaaren für die Zeitschliessung geringer. Dies letztere Resultat würde übrigens nach Eucken's Rechnung der Nernst'schen Reiztheorie (s. unten) entsprechen. Jedenfalls sind diese Ergebnisse nicht genügend, um eine prinzipielle Diskontinuität zwischen der Reizung mit konstantem Strom und mit hohen Kondensatorkapazitäten zu beweisen.

Von Interesse erscheint noch die wirkliche Grösse der in der Fig. 2 mit $A D$ bezeichneten Anfangsordinate, welche die kleinste zur indirekten Muskelreizung erforderliche Anfangsenergie darstellt. Sie wird höchstens ein wenig kleiner sein $(A R)$, wenn statt 1 Mf. ein konstanter Strom verwendet wird. Eigentlich muss man von einer Energiedichte im Nerven sprechen, d. h. die kleinste Energie durch den Nervenquerschnitt dividieren. Hier genügt es, aus den Versuchen mit 1 Mf. oder konstantem Strom die kleinste Stromstärke zu entnehmen, was leicht ist, soweit die Widerstände angegeben sind. In meinen Versuchen (dies Archiv Bd. 111, S. 542-546) betrug diese Stromstärke $10-25 \cdot 10^{-7}$ Amp.; nur einmal ging sie auf 5,5 herab und einmal auf 33 hinauf. In dem eingangs dieser Arbeit angeführten Versuch von $L$ apicque war sie $15 \cdot 10^{-7}$, in dem oben S. 183 mitgeteilten Versuch von Adam $34 \cdot 10^{-7}$ Amp. Es ist bemerkenswert, dass diese Mindeststromstärke, welche doch nach Streckenlänge, Nervendicke, Erregbarkeit sebr variieren muss, trotzdem eine so übereinstimmende Grössenordnung aufweist. Bei abgekürzter Entladung muss sie aber selbstverständlich stark vergrössert sein; in der Tat liegt sie in unseren Versuchen mit nur 0,1 $\sigma$ Entladungszeit zwischen 100 und $235 \cdot 10^{-7}$ Amp.

Schliesslich sei noch bemerkt, dass man sich den wirklichen Sachverhalt schwerlich so einfach vorstellen darf, wie die rohe Schematisierung der Fig. 2 ihn darstellt. Insbesondere wird gewiss zwischen Wirksamkeit und Unwirksamkeit des elektrisehen Vorgangs keine so harte und plötzliche Zeitgrenze existieren, wie es nach den Lapicque-Versuchen scheint, und wie es demgemäss in Schema durch den Absturz $E B$ dargestellt ist. Eine einfache Überlegung ergibt, dass die Lapicque-Versuche nicht entscheiden können, ob die Wirksamkeit des Vorgangs $D E T$ ganz plötzlich gemäss $E B$ aufhört oder allmählich abfällt.

Von einigem Interesse erscheint noch ein Blick auf die absoluten Werte des Energiebedarfs. In meiner vorigen Arbeit lag 
derselbe für das Optimum der Kapazität $\mathrm{zwischen} 0,0013$ und $\left.0,017 \mathrm{Erg}^{1}\right)$, die grösseren Werte im allgemeinen für grösseren Widerstand des Entladungskreises gültig. In den neuen Versuchen lag der Bedarf in der grossen Mehrzahl der Versuche zwischen 0,02 und 0,07 Erg; die Abkürzung der Entladung scheint also einen vermehrten Energiebedarf zu bedingen. Ungewöhnlich niedrige Zablen, nämlich weniger als 0,01 bis herab zu 0,0003 , zeigen sich regelmässig in den Versuchen mit sehr langer Nervenstrecke, und zwar unabhängig vom Gesamtwiderstand, der wie früher die Tendenz zeigt, den Bedarf etwas zu erhöhen.

\section{Bemerkungen zu einer Abhandlung von Hoo rweg.}

Obwohl nach den oben S. 175 gemachten kurzen Bemerkungen der Versuch von Lapieque für das Gebiet der Kondensatorreizung den Standpunkt Hoorweg's als völlig unhaltbar erwiesen hat, muss ich noch auf eine Abhandlung dieses Autors eingehen, welche ungemein schnell nach meiner letzten Arbeit über diesen Gegenstand erschienen ist ${ }^{2}$ ), wie gewöhnlich ohne jede Beibringung neuen experimentellen Materials. Hoorweg sucht meine Versuche, deren Ergebnisse sich seiner Theorie nicht fügen wollen, in nicht ernst zu nehmender Weise zu verdächtigen, indem er Dinge ins Feld führt, wie eine Selbstinduktion der Rheostaten (bei Widerständen von höchstens wenigen hundert Ohm!) oder Ungenauigkeit des von mir verwendeten Ed el mann'schen Präzisions- (Glimmer-) Kondensators oder gar den Umstand, dass ich die Nebenschliessungswiderstände $\beta$ nur nach ganzen $\mathrm{Ohm}$ und nicht genauer abgestuft habe; auf meine Versuche angewandt, bedeutet dies, dass ihm die Bestimmung der Potentiale bis auf $1 / 200$ - $^{1 / 500}$ Volt nicht genügt ${ }^{3}$ ). Er

1) Dieses Arch. Bd. 111 S. 559; die dort angegebenen Zablen sind mit 2 zu dividieren!

2) Dieses Arch. Bd. 114 S. 216. 1906.

3) Aus dem Umstande, dass in meinen Versuchen häufig das erforderliche Potential bei den hohen Kapazitäten sich nicht mehr merklich ändert, folgert Ho orweg seltsamerweise, dass diese Versuche "wenig Zutrauen verdienen“, während der allein richtige Schluss ist, dass der $p$-Bedarf bei steigender Kapazität sich einem Grenzwert asymptotisch nähert. Ein weiterer unbegreiflicher Irrtum Hoorweg's ist, dass die systematisch nach den böheren Kapazitäten zunehmende Abweichung der $p$-Werte von den nach seiner Theorie berechneten auf Ungenauigkeit der $p$-Beträge für hohe Kapazitaten beruhe. Im Gegenteil ist grade diese Dentung hier ofienbar ganz unmöglich. Endlich 
scheint ganz vergessen zu haben, dass er selbst sein Gesetz nur auf ganz grobe Versuche am Menschen gestützt hat, in welchen die Potentiale nach ganzen Volt variiert wurden, so dass die von mir hervorgehobenen systematischen Abweichungen von seinem angeblichen Gesetze kaum hervortreten konnten. Es ist mir sogar zweifelhaft, ob er überhaupt jemals an Froschpräparaten Versuche dieser Art angestellt hat; wie es scheint nicht, denn ohne die von ihm perhorreszierten Stöpselrheostaten wäre er kaum damit zustande gekommen.

Höchstens ein Punkt der Hoo rweg'schen Veröffentlichung scheint mir eine nähere Beleuchtung zu erfordern. Um seine Äusserungen zu begreifen, muss ich annehmen, dass er mich in einem wesentlichen Punkte völlig missverstanden hat. Ich habe vor 10 Jahren ${ }^{1}$ ) nachdrücklich darauf hingewiesen, dass die von $d u$ B o is-Reymond vorgenommene zeitliche Summation (Integration) der Erregungsdifferentiale wahrscheinlich für den Nerven seibst unzulässig, für ein träges Erfolgsorgan wie der Muskel aber, in welchem der Nerv chemische und mechanische Arbeit auslöst, unbedenklich zuzulassen ist, so dass für die Hervorrufung von Muskelzuckungen vom Nerven aus nicht mehr das einfache du B o is'sche Erregungsgesetz massgebend ist. Dies scheint nun Hoorweg dahin verstanden zu haben, dass eine einfache Stromesschwankung nach meiner Ansicht auf den Muskel überhaupt gar nicht erregend wirken soll, was mir durchaus fern $\operatorname{lag}^{2}$ ). Nun wird mir auf einmal klar, warum

ist es unrichtig, dass grade diese Werte auf die Berechnung der Konstanten $a$ und $b$ grossen Einfluss haben sollen; hätte Hoo rweg sich nur einmal die Mühe genommen, die zn dieser Berechnung dienenden Gauss'schen Formeln aufzustellen und zu betrachten, so hätte er gefunden, dass grade die $p$-Werte der hohen Kapazitäten auf die Berechnung der Konstanten den geringsten Einfluss häben. Dass Hoorweg eine aus guten Gründen nur für den Bereich zwischen grösster und optimaler Kapazität aufgestellte Formel deswegen "verdächtig“ findet, weil sie kein Optimum ergibt, wird niemand verstehen.

1) Dieses Arch. Bd. 75 S. 576. 1899. (Schon 1879 habe ich im Handbuch, Bd. 2 T. 1 S. 54, die Existenz einer Integralerregung für den Nerven bezweifelt.)

2) Nicht allein berechtigt keine einzige Stelle meiner Arbeiten dazu, mir diese Ansicht zuzuschreiben, sondern ich habe sogar die Erregung der Muskelsubstanz durch Stromesschwankungen vielfach ausdrücklich erwähnt (zuletzt Bd.111 S. 551 dieses Archivs); auch konnte doch Hoorweg wohl nicht annehmen, dass ich die Fortleitung der Erregung in der Muskelfaser mir prinzipiell anders denke als in der Nervenfaser, wo ich sie aus dem $\mathrm{d} u$ B o is 'schen Gesetze ableite. 
Hoorweg meinen wiederholten Hinweis, "dass die Erscheinungen der indirekten Muskelreizung nicht dem $d u$ Bo is'schen Gesetze folgen", "mit grosser Freude vernommen" hat, und warum er gegen meine vermeintliche Behauptung, dass die Stromesschwankung an sich nur für den Nerven und nicht für den Muskel ein Reiz sei, das vernichtende Argument schleudert: „es gibt nur eine Elektrizität" (!). Hätte Hoorweg meinen Satz (Bd. 111 S. 550) nur zu Ende gelesen, so hätte er sich sein Missverständnis erspart; denn es lautet unmittelbar weiter: „... und dass die von mir für den Nerven nicht zugelassene zeitliche Summation der Erregungen für das träge Organ des Muskels sehr wohl eintreten könnte". Schlimmer und noch weniger verzeihlich ist ein anderes gleichzeitiges Missverständnis. In meinem soeben zitierten Satze stehen zufällig hinter Muskelreizung die Worte „durch Kondensatorentladungen“, welche ebensogut hätten wegbleiben können; es handelte sich aber zufällig um diese Reizform. Hieraus macht nun Hoorweg, der unglaublich flüchtig gelesen haben muss, die Behauptung, das du Bois'sche Gesetz gelte zwar für andere Reize, aber nicht für. Kondensatorentladungen, und hat, es dann allerdings leicht, zu sagen, ein Gesetz, das für eine besondere Reizart nicht brauchbar ist, ist überhaupt nicht brauchbar.

Diese Divergenzen, an welchen meine vollkommen klare Darstellung unschuldig ist, hängen mit einer anderen mehr prinzipiellen Meinungsverschiedenheit zusammen. Hoorweg tadelt mich, dass ich beharrlich nicht von Versuchen und Gesetzmässigkeiten über Nervenreizung, sondern nur von solchen über indirekte Muskelreizung spreche. Und doch ist dies mehr als blosse berechtigte Vorsicht. Im Gegenteil, das Onus der Beweisfühung ruht auf Demjenigen, welcher behauptet, dass hier Erregungsgesetze des Nerven aufgedeckt werden. Wenn wie hier dureh irgendeine Verkettung wenig träger Elemente aus der Ferne auf ein träges Gebilde eingewirkt wird, so gibt allemal das letz tere den Ausschlag über Erfolg oder Nichterfolg, und seine Eigensehaften sind es, die das Experiment enthüllt. Ein grobes, aber vahe liegendes Beispiel wird genügen. Bei hinreichend frequenter Nervenreizung erhält man statt der Einzelzuckungen Tetanus; wer so urteilt wie Hoorweg, konnte dies vor 40 Jahren als ein Reizgesetz des Nerven betrachten, d. h. annehmen, dass der Nerv Reize oberhalb einer gewissen Frequenz nicht mehr trennen könne, sondern verschmelze. Das wäre ein falscher 
Schluss gewesen, denn Helmholtz hat 1864 durch den der Reizfrequenz entsprechenden Muskelton gezeigt, dass die Verschmelzung erst in der kontraktilen Substanz erfolgt. Bis ein Gegenbeweis erbracht ist, erscheint es als eine berechtigte und als die einfachste Annahme, dass alle Nervengattungen dasselbe Erregungsgesetz haben, wäbrend ganz verschiedene Gesetze vorgetäuscht werden, wenn man die Erfolge in den verschiedenen Endapparaten zum Massistabe nimmt. Gewiss wird doch auch $\mathrm{Hoorweg}$ annehmen, dass der Nerv eines besonders trägen, z. B. glattmuskeligen Organs einer besonders anhaltenden Einwirkung bedarf, um dies Organ zu reizen, und es für willkürlich halten, dem betreffenden Nerven obne Beweis ein besonderes Reizgesetz zuzuschreiben. Dass etwa jeder Nerv ein besonderes, seinem Endorgan angepasstes Erregungsgesetz habe, ist um so unwahrscheinlicher, als elektrische Reizung im Nervenverlauf ein ganz widernatürlicher Eingriff, wenu auch dem Physiologen sehr geläufig ist.

Der von Hoorw e $\mathrm{g}$ angeführte Umstand, dass auch für sensible Nerven mit Kondensatorversuchen dieselben Gesetzmässigkeiten erwiesen seien wie für motorische, würde hieran nichts ändern. Denn grade für die Zentralorgane, die hier den Erfolg bedingen, ist längst bekannt, dass sie vielfach erst auf summierte Reize ansprechen, also von Trägheitseigenschaften nicht frei sind. Auf diesen aber beruht es nach meiner Vorstellung, dass eine Ausdehnung der Einwirkungen auf eine gewisse Zeit Erfolgsbedingung ist. Absolut frei hiervon dürfte auch der Nerv nicht sein, aber hier ist der Zeiteinfluss kaum nach weisbar.

Natürlich babe ich nicht unterlassen, mich zu vergewissern, ob in der Tat bei direkter Muskelreizung durch Kondensatorentladungen sich dieselben Beziehungen zeigen wie bei indirekter. Aus den Versuchen teile ich folgende Beispiele mit. Der Gastroknemius eines kurarisierten Frosches wirkte auf den Zuckungstelegraphen. Die Versuchsanordnung war genau die in meiner letzten Arbeit verwendete. Auch die Buchstaben haben die dort angegebene Bedeutung. 
1. Beispiel.

\begin{tabular}{|c|c|c|c|c|c|c|}
\hline \multirow{2}{*}{$\begin{array}{c}\text { Kapazität } \\
\text { in } \\
\text { Mikro- } \\
\text { farad } \\
c\end{array}$} & \multirow{2}{*}{$\begin{array}{c}\text { Spannung } \\
\text { der } \\
\text { Kette } \\
v\end{array}$} & \multicolumn{2}{|c|}{ Widerstand } & \multicolumn{3}{|c|}{ Also war erforderlich } \\
\hline & & $\begin{array}{c}\text { des Haupt- } \\
\text { kreises } \\
\alpha\end{array}$ & $\begin{array}{c}\text { der Neben- } \\
\text { schliessung } \\
\beta\end{array}$ & $\begin{array}{c}\text { die } \\
\text { Spannung } \\
p\end{array}$ & $\begin{array}{c}\text { die } \\
\text { Elektrizi- } \\
\text { tätsmenge } \\
p c\end{array}$ & $\mid \begin{array}{c}\text { die } \\
\text { (doppelte) } \\
\text { Energie } \\
p^{2} c\end{array}$ \\
\hline $\begin{array}{l}1,0 \\
0,5 \\
0,2 \\
0,1 \\
0,05\end{array}$ & $\begin{array}{lc}2 & \text { Volt } \\
2 & " \\
2 & " \\
4 & " \\
4 & "\end{array}$ & $\begin{array}{l}10 \mathrm{Ohm} \\
10 " \\
10 " \\
10 " \\
10 "\end{array}$ & $\mid \begin{array}{rl}12 & \mathrm{Ohm} \\
22 & " \\
200 & " \\
21 & " \\
25000 & "\end{array}$ & $\begin{array}{l}1,091 \text { Volt } \\
1,375 ~ " \\
1,905 \quad " \\
2,700 \% \\
4,000 "\end{array}$ & & $\begin{array}{l}0,945 \\
0,726 \\
0,739 \\
0,800\end{array}$ \\
\hline
\end{tabular}

2. Beispiel. (Mit A. A d a m.)

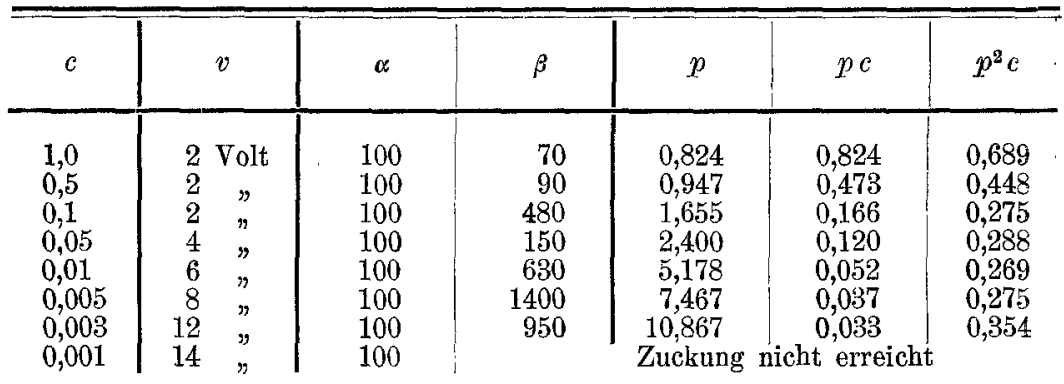

Diese beiden Beispiele werden genügen, zu zeigen, dass das bemerkenswerteste von $\mathrm{Hoorweg}$ mit indirekter Reizung gefundene Verhalten, dass nämlich die Energie bei einer gewissen Kapazität ein Minimum durchläuft, auch für direkte zutrifft. Dass es sich hier um eine Eigenschaft des Muskels und anderer träger Organe handelt, wird man also so lange annehmen dürfen, bis dasselbe Gesetz für blosse Nervenreizung bewiesen ist.

Sicher haben alle erregbaren Organe, also auch Nerv und Muskel, gewisse gemeinsame Eigenschaften. Das elementare Gesetz der elektrischen Reizung dürfte für beide prinzipiell dasselbe sein und sein einfachster Ausdruck die du B o is' sche Formel mit dem von mir hinzugefügten negativen Vorzeichen, welches die polaren Beziehungen ausdrückt. Ich habe gezeigt, dass, dieses Gesetz unter Berücksichtigung der Polarisierbarkeit der Fasern und der genau wie Selbstinduktion wirkenden Aktionsströme eine exakte Erklärung der Erregungsleitung gestattet, und betrachte es daher nach wie vor als das wirkliche elementare Erregungsgesetz für den Nerven und selbstverständlich auch für den Muskel. 
Zwar hat Hoor weg gegen meine Theorie der Erregungsleitung Einwände erhoben ${ }^{1}$ ), ich habe aber gezeigt, dass dieselben völlig unzureichend sind ${ }^{2}$ ). Hoorweg selbst vertritt noch immer die sogenannte Kabeltheorie der Leitung, d. h, die Ableitung von blossen Kernleitereigenschaften (Kapazität oder Polarisation); ich babe aber schon $1873^{3}$ ) und besonders $1905^{4}$ ) darauf hingewiesen, dass die für diesen Fall geltende Differentialgleichung (Wärme- oder Diffusionsgleichung) niemals eine Fortpflanzungsgeschwindigkeit von der Grössenordnung der nervösen oder gar der muskulären ergeben kann. Dies wird erst möglich durch das Hinzukommen eines der Selbstinduktion analogen Moments, und dieses liegt eben im Gesetz der Aktionsströme.

Dies letztere Moment wird in neueren Ausführungen Ho or w eg's ${ }^{5}$ ) von neuem übersehen. Im Anschluss an die Nernst'sche Theorie gibt er, wie in früheren Publikationen, weit ausblickende Aperçus, in welchen man u. a. überrascht erfährt, dass es "eine alte, noch nicht gelöste Streitfrage " ist, ob es einen Unterschied zwischen den elektrotonischen und den Aktionsströmen gibt! Ich werde auf diese Dinge nicht eingehen, obwohl ich Grund hätte, ihm Nichtbeachtung gewisser Arbeiten nachzuweisen. Aber eine andere bei dieser Gelegenheit von ihm gemachte Bemerkung ${ }^{6}$ ) fordert zum Widerspruch heraus. Er sagt: „Bekanntlich können nach den Untersuchungen Macdonald's, Oker-Blom's und Bernstein's die eigenen Ströme der Muskeln und der Nerven als wahre Konzentrationsströme betrachtet werden." Wer dies liest, muss annehmen, dass die genannten Autoren eine begründete und klare Ableitung der tierischelektrischen Vorgänge aus dem Prinzip der Konzentrationsketten geliefert haben. Einer solchen Behauptung werden diese zum Teil selbst auf das entschiedenste widersprechen. So beachtenswert und natürlich auch die Bemühungen der Genannten und anderer sind, die neu gewonnenen Erkenntnisse der Elektrochemie auf diesem Gebiete zu verwerten, so wenig ist bisher ein entschiedener Erfolg zu verzeichnen, ja, es stehen sogar grosse Bedenken entgegen, ins-

1) Dieses Arch. Bd. 85 S. 106f. 1901; Bd. 114 S. 222.1906 usw.

2) Dieses Arch. Bd. 86 S. 103 f. 1901, und besonders Bd. 109 S. 129 f. 1905.

3) Handb. d. Physiol. Bd. 2 Teil 1 S. 195.

4) Dieses Arch. Bd. 109 S. 104 ff.

5) Dieses Arch. Bd. 119 S. 412 ff. 1907.

6) A. a. 0. S. 416.

E. Pflüger, Archiv für Physiologie. Bd. 12\%. 
besondere wenn man die berechtigte Forderung stellt, dass jedes Erklärungsprinzip für die Ruheströme zu verwerfen ist, wenn es nicht auch die Aktionsströme erklärt ${ }^{1}$ ). Vielleicht wird man doch auf eine physikalische Erklärung des von mir aufgestellten Satzes, dass alle Protoplasmen auf partielles Absterben und auf partielle Erregung mit Negativität des alterierten Anteils reagieren, oder etwas Befriedigenderes, länger warten müssen, als $\mathrm{Hoorweg}$ es sich denkt.

\section{Die Nernst'sche Theorie der elektrischen Reizung.}

Die Physiologie kann es nur dankbar begrüssen, wenn hervorragende Physiker sich einem ihrer Probleme zuwenden, und nur sehr ungern, aber notgedrungen, entschliesse ich mich, gegen Nernst's Theorie der elektrischen Reizung ${ }^{2}$ ) einige Einwände zu erheben. Es ist kein Vorwurf, sondern durchaus begreiflich, dass Nernst eine Anzahl hier mitsprechender physiologischer Tatsachen anscheinend nicht kannte oder (wie das "Hineinschleichen in den Strom ${ }^{\text {}}$ ) erst nach Aufstellung seiner Theorie erfuhr, noch weniger dass die auf unorganischem Gebiete sieggewohnte Physik geneigt ist, die Schwierigkeiten zu unterschätzen, welche die organisierte Materie der physikalischen Durchleuchtung entgegenstellt, und welche dem Physiologen von Fach nur allzusehr sich aufdrängen.

Die Grundlage der Theorie ist folgendes:, Die organisierten Gewebe sind elektrolytische Leiter, folglich kann der elektrische Strom in ihnen keine anderen Wirkungen entfalten als Ionenverschiebungen, d. h. Konzentrationsänderungen; also müssen letztere die Ursache des physiologischen Effektes sein. Aus der berechneten Grösse der Konzentrationsänderung für verschiedene Arten des Stromverlaufes (konstanter Strom, Wechselströme, Kondensatorentladungen) leitet Nernst quantitative Reizgesetze ab, welche er durch das physiologische Versuchsmaterial bestätigt findet.

Für den konstanten Strom muss er freilich sofort eine Hilfshypothese machen, da die Theorie sonst selbst bei der langsamsten Stromzunahme eine Erregung ergeben würde; er nimmt daher an,

1) Auch in den diesen letzteren Gegenstand betreffenden, sehr weitgehenden .neueren Hypothesen von Höber und Macd onald, auf welche ich hier natürlich nicht eingehen kann, wird niemand eine befriedigende Lösung der Frage, sondern höchstens die Andeutung entfernter Möglichkeiten erblicken.

2) Siehe die für Physiologen bestimmte Darstellung in diesem Archiv Bd. 122 S. 275. 1908. 
dass die Konzentrationsänderung in der Protoplasmahaut irgendeinen chemischen Umsatz auslöst, der die Reizschwelle erhöht, d. h. eine grössere Konzentrationsänderung zur Reizung erforderlich macht; diese "Akkommodation" an den Strom werde um so grösser, je langsamer die Konzentrationsänderung erfolgt. Die Akkommodation darf offenbar nicht, wozu manche geneigt sind, als etwas Physiologisches, etwa eine durch Abstumpfung oder Ermüdung entstehende Erregbarkeitsverminderung aufgefasst werden; denn damit würde ja der Anspruch aufgegeben sein, ein Reizgesetz auf rein physikalischchemischer Grundlage zu liefern. Vielmehr sagt $\mathrm{N}$ e r n st ganz deutlich, dass er sich hier einen "nach den Gesetzen der chemischen Reaktionsgeschwindigkeit" sich abspielenden reaktiven Vorgang denkt, dessen nähere Erkenntnis er von der Zukunft erwartet. Vorläufig veranschaulicht er nur die Wirkung der Akkommodation durch eine mathematische Formulierung, auf welche ich weiter unten eingehen werde.

Nernst bemerkt selbst (S. 307, 308), dass es für die von ihm ausgeführte Rechnung auf dasselbe hinausläuft, ob man die Reizung auf Konzentrationsänderung oder auf galvanische Polarisation zurückführt; dies letztere ist aber in der Physiologie schon längst geschehen. Seit Pflug er die elektrische Reizung auf Entstehung von Katelektrotonus oder Verschwinden von Anelektrotonus zurückgeführt hat und ich selbst den Elektrotonus als eine Polarisation all der Grenze der Protoplasma- und der Hüllensubstanz der Nervenfaser erklärt babe, war es klar, dass man die Bedingung der elektrischen Reizung so ausdrücken konnte, wie ich es 1873 zuerst getan habe ${ }^{1}$ ), nämlich als eine Veränderung der Polarisation an der Protoplasmagrenze in (absolut) negativem Sinne. Dass sich auch die von N ernst behandelte Konzentrationsänderung nur auf diese Fläche beziehen kann, ist sicher und aus seinen eigenen Worten (S. 277) zu entnehmen. Nernst würde auch zweifellos, wenn ihm das Pflüger'sche polare Erregungsgesetz ${ }^{2}$ ) bekannt wäre, dasselbe leicht in seine Theorie einfügen können, sei es durch die Annahme, dass nur Abnahme oder nur Zunahme der Konzentration ein Reiz ist, oder durch die andere, dass nur Konzentrationsände-

1) Dieses Arch. Bd. 7 S. 364. 1873.

2) Der Strom reizt bei seiner Schliessung oder Zunahme nur an der Kathode, bei der Öffnung oder Abnahme nur an der Anode. 
rungen bestimmter Natur in Betracht kommen. Insofern ist aber die Nernst'sche Theorie etwas Neues, als zum ersten Male versucht wird, auf Grund des Zusammenhanges der Erregung mit der Polarisation quantitative Reizgesetze aufzustellen ${ }^{1}$ ).

Und doch muss vom physiologischen Standpunkt ein wesentlicher Einwand erboben werden. In der oben fast wörtlich angeführten Begründung der Theorie wird nämlich nicht berücksichtigt, dass das Protoplasma zwar die Eigenschaften eines elektrolytischen Leiters hat, aber ausserdem noch zahlreiche andere, von denen viele über das gegenwärtig physikalisch Begreifbare weit hinausgehen; darüber sind auch diejenigen Physiologen einig, welche dem sogenannten Vitalismus vollkommen abhold sind, d. h. ein Ignorabimus auf materiellem Gebiete nicht unterschreiben.

Eine solche Figenschaft des Protoplasmas, von welcher Nernst anscheinend keine Notiz nimmt, die aber hier sicher sehr in Betracht gezogen werden muss, ist dessen spezifische elektromotorische Reaktion, der Aktionsstrom. Es ist durch die Arbeiten von d u Bois-Reymond, Bernstein und mir auf das bestimmteste nachgewiesen, dass an der Reizstelle die Faser den Reiz unverzüglich mit einer sehr kräftigen ${ }^{2}$ ) Potentialdifferenz der gereizten Stelle gegen die unerregte Kontinuität beantwortet, und dass diese Potentialdifferenz sich an jeder Faserstelle wiederholt, welche von der wellenförmig ablaufenden Erregung passiert wird. Diese Wirkung darf aber offenbar bei der infinitesimalen Behandlung des Vorganges an der Reizstelle ebensowenig unberücksichtigt bleiben, wie die von Nernst allein berücksichtigte diffusorische Rückwirkung. Dieser Einwand gegen die Ableitung des Nernst'schen Gesetzes könnte nur dadurch beseitigt werden, dass etwa nachgewiesen würde, dass die diffusorische Rückwirkung eine Geschwindigkeit von unvergleich-

1) Die hier geltende Differentialgleichung (Diffusions- oder Wärmegleichung) habe auch ich in einer Arbeit, welche mit der Nernst'schen einige Berührungspunkte hat, integriert (dieses Arch. Bd. 109 S. 104 ff. 1905). Jedoch betraf diese Arbeit eine wesentlich andere Aufgabe, nämlich die Etablierung der stationären Polarisation durch einen Danerstrom (Elektrotonus).

2) Die nach aussen ableitbaren elektromotorischen Kräfte stellen wegen der inneren Schliessung nur einen, vielleicht sehr kleinen Bruchteil der wirklichen dar, und letztere bewirken wegen der Kleinheit der Widerstände in den mikroskopischen Dimensionen zweifellos sehr starke Lokalströme. (Vgl. mein Handb. . Physiol. Bd. 2 Teil 1 S. 194. Leipzig 1879.) 
lich höherer Grössenordnung hat als die Entwicklung des Aktionsstromes, wofür vor der Hand nichts spricht; jedenfalls erstreckt sich die Nernst'sche Integration und ihre Anwendung auf Zeiten, innerhalb deren die Aktionsstromwelle bereits beträchtliche Strecken zurücklegen kann.

Sobald aber Nernst diesen Umständen Rechnung trägt, wird er möglicherweise $\mathrm{zu}$ ähnlichen Betrachtungen und rechnerischen Ergebnissen gedrängt werden wie ich, als ich die wellenartige Erregungsleitung auf die Polarisierbarkeit in Verbindung mit der elektromotorischen Reaktion zurückzuführen suchte, wobei man statt der Diffusionsgleichung auf die sogenannte Telegraphengleichung geführt wird ${ }^{1}$ ). Überhaupt lässt sich nach meiner schon 1879 ausgesprochenen Überzeugung das Problem der lokalen Reizung von demjenigen der wellenartigen Fortleitung nicht trennen ${ }^{2}$ ), und ebensowenig das Gesetz der elektrisehen Reizung von demjenigen anderer, z. B. mechanischer Reizungen. Hier zeigt sich womöglich noch deutlicher als dort die massgebende Bedeutung der Plötzlichkeit der Veränderung: man kann bekanntlich einen Nerven sehr allmählich zerquetschen, ohne ihn zu reizen, während der leiseste Stoss ihn reizt; schwerlich aber wird man annehmen können, dass etwas der Nernstschen Akkommodation Analoges bei jener Wirkungslosigkeit im Spiele ist.

Das Ergebnis der Rechnung von Nernst lässt sich kurz dahin formulieren, dass, soweit die Akkommodation fnicht modifizierend eingreift, in jedem der drei untersuchten Fälle (s. oben) ein von den Versuchsvariablen unabbängiges Energiequantum für das Zustandekommen der Minimalzuckung erforderlich ist. Dass Nernst sein Ergebnis nicht so ausdrückt, hat wohl darin seinen Grund, dass er, wie schon erwähnt, das polare Erregungsgesetz nicht berücksichtigt. Nach diesem muss bei Wechselströmen die Reizung zwischen beiden Elektroden alternieren ${ }^{3}$ ), so dass bei einem Sinusstrom jede

1) Vgl. dieses Arch. Bd. 75 S. 475. 1899, Bd. 109 S. 111, Bd. 110 S. 254.1905.

2) Vgl. das zitierte Handb. a. a. O. S. 193.

3) Dies gilt auch für sogenannte unipolare Reizung, d. h. für denjenigen Fall, in welchem nur eine Elektrode am Nerven, die andere an irgendeinem anderen Teil des Präparats oder Körpers liegt. Bekanntlich ist dann die zweite Elektrode auf viele Nervenstellen verteilt und dadurch ihre Stromdichte so vermindert, dass für die Reizschwelle nur die am Nerven selbst liegende Ellektrode, also auch nur die eine der beiden Stromrichtungen in Betracht kommt. 
zwischen zwei Kulminationen liegende Strecke als eine neue Reizperiode zu betrachten ist. Ist nun die Stromstärke $i=J \sin n t$, worin $n$ die Schwingungszahl für $2 \pi$ Sekunden, so ist die Energie einer solchen Periode

$$
E=w J_{-\frac{\pi}{2 n}}^{\frac{\pi}{2 n}} \sin ^{2} n t d t=\frac{\pi u}{2} \cdot \frac{J^{2}}{n} .
$$

Wenn also die Rechnung von Nernst ergibt $\frac{J}{\sqrt{n}}=$ const. oder $\frac{J^{2}}{n}=$ const., so ergibt sie eben, dass zur Minimalreizung die auf jeden Einzelreiz fallende Energie massgebend ist ${ }^{1}$ ). Für die Zeitschliessung und die Kondensatorentladung ist selbstverständlich das von Nernst resp. Eucken ${ }^{2}$ ) gefundene Gesetz $i \sqrt{t}=$ const. resp. $p \sqrt{c}=$ const. identisch mit $w i^{2} t=$ const. resp. $\frac{p^{2} c}{2}=$ const., d. h. der Energiebedarf ist konstant.

Nernst und Eucken finden ihr Rechnungsresultat in hinreichender Übereinstimmung mit den Versuchen. Ich vermeide es, in dieser Beziehung auf diejenigen Reizarten einzugehen, über die ich selbst keine Versuche angestellt habe; aber für Kondensatorentladungen trifft Eucken's Formel sicher nicht zu, wie z. B. ein Blick auf die enorm variierenden Energiewerte in der letzten Kolumne meiner zahlreichen Versuchstabellen (Bd. 111 S. 542-546) überzengend dartut. Eucken konnte dies natürlich nicht entgehen; er findet aber die Konstanz der Energiebeträge "wenigstens innerhalb eines gewissen Gebietes" bestätigt; ich kann aber selbst diese eingeschränkte Behauptung nicht zugeben; auch in sehr kleinem Bereich treten nur deshalb bei Eucken die Abweichungen von der Konstanz weniger hervor, weil bei ihm nur die Quadratwurzeln der Energiezahlen figurieren; auch führt er z. B. in dem meiner Arbeit entnommenen Versuch nicht die vollständige Reihe an. Selbstverständlich kann der jetzt von uns gefundene Umstand, dass bei $a b$ -

1) Für die Zeit von $n$ Schwingungen, also für $2 \pi$ oder für 1 Sek, ist natürlich die Energiesumme nur von $J^{2}$ abhängig. Für die Reizschwelle des Tetanus kommt es aber offenbar, da eine Summation der Einzelreize höchstens im Beginn stattfindet, nur auf die Stärke des Einzelreizes an.

2) Sitzungsber. d. Preuss. Akad. 1908 S. 524 ff. (Unsere Arbeit war schon im wesentlichen abgeschlossen, als diese Abhandlung erschien.) 
gebrochenen Entladungen ein annähernd konstanter Energiebedarf sich herausstellt, nicht als Bestätigung der Theorie gelten.

Besonders gross sind die Abweichungen bei den höchsten und bei den niedrigsten Kapazitäten, und mit diesem Umstande sucht Eucken sich abzufinden. Dass die kleinen Kapazitäten hohe Energiebeträge erfordern, könne vielleicht aus Deformationen des Stromverlaufs durch Selbstinduktion usw. erklärt werden; jedoch hält er auch physiologische Ursachen für nicht ausgeschlossen. Für die Abweichungen bei den grösseren Kapazitäten aber zieht er die Akkommodation zur Erklärung heran, welche ja um so mehr sich geltend macht, je länger der Stromverlauf sich hinzieht. Ja, Eucken will sogar das eingangs dieser Arbeit erörterte Lapicquesche Resultat auf diesem Wege deuten: Die Nutzzeit ist nach ihm diejenige Zeit, innerhalb deren die Akkommodation noch nicht den Reizeffekt vereitelt ${ }^{1}$ ).

Hier stellen sich aber sehr erhebliche Schwierigkeiten ein, welche nur dann klar übersehbar sind, wenn man versucht, der Wirkung der Nernst'schen Akkommodation etwas näher zu treten. Hierzu ist der einzige Anhaltspunkt die von Nernst (S. 281) aufgestellte Formel 7. Nach Nernst muss die Konzentrationsänderung $c-c_{0}$, um zu reizen, einen bestimmten (konstanten) Betrag $A$ überschreiten, also $c-c_{0}>A$ sein. Die Akkommodation bewirkt nun, dass statt $A$ der Betrag $A\left(1+B \int_{0}^{t}\left(c-c_{0}\right) d t\right)$ überschritten werden muss. Wir dürfen hierin, wenn wir $c$ vorübergehend als die bei der Minimalreizung grade erreichte Konzentration ansehen, das Zeichen $>$ durch $=$ ersetzen, so dass die wirkliche Bedingung für Minimalreizung lautet (für $A B$ wollen wir die Konstante $R$ einführen):

$$
c-c_{0}=A+R \int_{0}^{t}\left(c-c_{0}\right) d t . . . .
$$

Der Ausdruck rechts kommt $\boldsymbol{A}$ um so näher, d. h. die Akkommodation ist um so unmerklicher, je kleiner $t$ und die Konstante $R$.

1) Eucken berechnet aus dem Lapicque'schen Versuch die Relativwerte der am Ende der Nutzzeit erreichten Konzentration (Spalte 6 seiner Tabelle, S. 539). Die Zahlen zeigen einen sehr ähnlichen Gang wie die (nicht etwa ihnen proportionalen) Quadratwurzeln unserer $E^{*}-$ Werte in demselben Versuch (s. oben S. 174); wie diese durchlaufen sie ein Minimum bei $0,02 \mathrm{Mf}$. 
Wenn man nun die Gleichung (5) nach $t$ differentiiert $^{1}$ ) und die entstehende Differentialgleichung unter der Grundbedingung integriert, dass für $t=0 \quad c-c_{0}=A$ sein soll, so erhält man als eine neue Form der Reizbedingung ${ }^{2}$ ):

$$
c-c_{0}>A e^{R t} \quad \text { oder } \quad\left(c-c_{0}\right) e^{-R t}>A . \quad .
$$

Zunächst möchte ich darauf aufmerksam machen, dass diese Reizbedingung sehr an die Formel erinnert, durch welche Engelmann und nach ihm Hoorweg darstellen wollten, dass der Strom nur bei seinem Entstehen reizt; sie setzten nämlich den Reizwert der Stromstärke $i$ proportional dem Ausdruck $i e^{-\beta t}$ (worin $\beta$ eine Konstante); nur dass hier $c-c_{0}$ nicht die Stromstärke selbst, sondern eine Funktion derselben ist.

Mittels (6) kann man aber die Wirkung der Akkommodation für den konstanten Strom vollkommen übersehen. Nach Nernst (S. 288, 300) ist nämlich hier die Konzentrationsänderung durch die Stromstärke $i$ zur Zeit $t$ :

$$
-\left(c-c_{0}\right)=\nu i \sqrt{\frac{t}{\pi k}}(>A), \cdot \ldots . .
$$

worin $v$ und $k$ Konstanten sind. Setzt man dies in (6) ein, so erhält man (das Vorzeichen liommt hier nicht in Betracht) als Reizbedingung für eine Zeitschliessung:

$$
v i \sqrt{\frac{t}{\pi \bar{k}}} \cdot e^{-R t}>A ; . . . \quad . \quad . \quad
$$

für Minimalzuckungen müsste also infolge der Akkommodation nicht $i \sqrt{\bar{t}}=$ const., sondern

$$
i \sqrt{t} \cdot e^{-R t}=\text { const. . . . . . . . }
$$

sein. Wenn nun Nernst in den Versuchsreihen von G. Weiss 'und $\mathrm{L}$ apicque, welche bis $t=3 \sigma$ reichen, sein Quadratwurzelgesetz $i \sqrt{t}=$ const. hinreichend bestätigt findet, so kann dies nur auf einer ausserordentlichen Kleinheit der Konstante $R$ beruhen, worauf wir noch zurückkommen.

1) Dies wäre bei $>$ statt $=$ unzulässig gewesen.

2). Hier und im folgenden kann man, wenn man will, überall $=$ statt $>$ setzen; die Ausdrücke gelten dann für den Schwellenwert der Konzentrationsänderung. 
Eine weitere sich aufdrängende Folgerung aus der Nernst'schen Formulierung der Akkommodation ist für Zeitschliessungen folgende. Die linke Seite von (8) erreicht für $t=\frac{1}{2 R}$ ein Maximum, nämlich

$$
\frac{\nu i}{\sqrt{2 R \pi k}} \cdot e^{-\frac{1}{2}}
$$

und zum mindesten muss, damit der Strom reizen kann, dies Maximum $>A$ sein; hieraus folgt aber, dass eine Stromstärke $i$ niemals reizen kann, wenn nicht

$$
i>\frac{A}{\nu} \sqrt{2 R \pi k e} . \text {. . . . . . . }
$$

Diese Folgerung lässt sich aber sehr gut an den Versuchen prüfen. In dem von Nernst S. 309, Tab. IX angeführten Versuch von G. Weiss ist z. B., wenn man auf die Einheiten des Originals ${ }^{1}$ ) zurückgeht, für die kürzeste und die längste Schliessungsdauer $i \sqrt{t}=0,536 \cdot 10^{-7}$ Amp. $\sqrt{\text { sek, }}$, welcher Betrag nach $(7)=\frac{A}{\nu} \sqrt{\pi k}$ sein muss. Nach (10) muss also, um überhaupt reizen zu können;

$$
i>\sqrt{2 \operatorname{Re~sek}} \cdot 0,536 \cdot 10^{-7} \text { Amp. . . . . }
$$

sein. Hierin ist nur noch $R$ unbekannt. Um dies zu bestimmen, ist der soeben erwähnte Versuch ungeeignet, weil in ihm $i \sqrt{t}$ ein Minimum durchläuft. Nach der Akkommodationstheorie ist vielmehr zu erwarten, dass wegen (8) das erforderliche $i \sqrt{t}$ um so grösser wird, je länger $t$. Dies zeigt sich nun freilich, was bedenklich machen muss, nur in einem Teil der Versuche, aber einigermassen in dem von Nernst als Tab. $X$ wiedergegebenen Weiss'schen Versuch, in welchem $i \sqrt{t}$ bei der kürzesten Zeit 370 , bei der längsten (von 0,003 sek) 487 beträgt. Nach (9) müsste also ungefähr

$$
e^{-R .0,003 \mathrm{selk}}=\frac{370}{487}
$$

sein, woraus sich berechnet $R=91$ sek $^{-1}$. Setzt man nun diesen Betrag in (11) ein, so ergibt sich als Grenze der noch reizfähigen Stromstärke

$$
i>11,94 \cdot 10^{-7} \text { Amp., }
$$

was überraschend gut zu den oben S. 201 angeführten Resultaten stimmt; freilich ist leicht einzusehen, dass diese Übereinstimmung ein Zufall sein kann und gegenüber den Bedenken der Theorie wenig bedeutet.

1) Arch. ital. d. biol. Bd. 35 S. 434. 1901. 
Auch für Wechselströme lässt sich leicht die Reizbedingung so gestalten, dass sie der Akkommodation, d. h. der Nernst'schen Gleichung (7) (hier 5) Rechnung trägt. Hier ist für den Strom $i=J \sin \left(n t+\frac{\pi}{2}\right)$ nach Nernst (S. 285) die Konzentrationsänderung

$$
c-c_{0}=\frac{\nu J}{\sqrt{n \bar{k}}} \sin \left(n t+\frac{\pi}{4}\right), \ldots . . .
$$

welcher Ausdruck also, mit $e^{-R t}$ multipliziert, $>A$ sein muss, wobei $t$ jedesmal vom Beginn eines Einzelreizes zu rechnen ist. Da aber dieses Produkt ein Maximum durchläuft, so kann man jedenfalls dieses Maximum $>A$ setzen, wodurch die Zeit herausgeschafft wird. So findet man, dass für Minimalreizung

$$
\nu J \sqrt{\frac{n}{k\left(n^{2}+k^{2}\right)}} \cdot e^{-\frac{R}{n}\left(\operatorname{arctg} \frac{n}{k}-\frac{\pi}{4}\right)}=\text { const. . . . }
$$

sein muss, was beträchtlich vou dem einfachen Quadratwurzelgesetz $\frac{J}{\sqrt{n}}=$ const. abweicht. Diese Abweichung ist um so grösser, je kleiner die Wechselzahl $n$, und es wird schwerlich mit der Nernstschen Formulierung der Akkommodation zu vereinbaren sein, dass Nernst das einfache Quadratwurzelgesetz bis zu einer Wechselzahl von nur 5,3 (S. 291) ziemlich bewährt findet, d. h. für eine Dauer der (halben) Periode von 0,094 sek oder $94 \sigma$.

Für Kondensatorentladungen konnte ich leider die Einführung dér Akkommodationsgleichung (6) nicht so direkt vornehmen wie für konstante und Wechselströme, weil Eucken hier für $c-c_{0}$ keine geschlossene Formel gibt, sondern von Äusdrücken, welche die Zeit enthalten, nur Doppelintegrale; der Faktor. $e^{-R t}$ liess sich infolgedessen nicht einführen ${ }^{1}$ ), wozu jedoch Eucken wohl einen Weg finden würde.

Zunächst seien einige interessante Rechnungsresultate Eucken's angeführt. Bei Kondensatorentladungen muss natürlich die Konzentration ein Maximum durchlaufen; nach Eucken wird dasselbe erreicht, wenn das Potential auf den 2,35ten Teil seines Anfangswertes gesunken oder $81,9 \%$ der vorhandenen Energie verausgabt sind. Für 1 Mf. ist dies erst 0,06 sek $(60$ o) nach dem Beginn der Fall,

1) Der von Eucken gegebene geschlossene Ausdruck für das Maximum und dessen Zeit ist für diesen Zweck nicht verwendbar. 
für 0,1 Mf. nach $6 \sigma$, für 0,01 Mf. nach $0,6 \sigma$ usf. Ferner erfordert eine bestimmte Konzentration, wenn sie einmal durch Zeitschliessung, einmal durch Kondensatorentladung erreicht wird, im letzteren Fall einen $1,71 \mathrm{mal}$ so grossen Energieaufwand als im ersteren; diese Verbältniszahl sinkt auf 1,40 , wenn nur der bis zur Erreichung des Maximums entladene Teil der Kondensatorenergie in Betracht gezogen wird. Hiernach ist zu erwarten, dass bei gleich lange dauerndem Schwellenreiz die Kondensatorentladung mehr Energieaufwand beansprucht als die Zeitschliessung; dass dies sich in Versuchen Lapicque's bestätigt, ist schon oben S. 201 erwähnt.

Dagegen scheint mir ein unlösbarer Widerspruch zu bestehen zwischen der Eucken'schen Deutung des Lapicque'schen Versuches und dem, was oben über die Wirkung der Akkommodation bei Zeitschliessungen gefolgert worden ist. Bei letzteren ist bis zu Zeiten von $3 \sigma$ noch keine wesentliche Einmischung der Akkommodation zu merken, bei Wechselströmen sogar bis $94 \sigma$; bei Entladung einer Kapazität von 1 Mf. dagegen soll- sie; obwohl hier die Konzentration bis $60 \sigma$ anwächst, schon nach $1,81 \sigma$ das weitere Wachstum effektlos machen; zu dieser Zeit ist der Faktor $e^{-R t}$, mit welchem die Konzentrationsänderung zu multiplizieren ist, bei der oben ermittelten Grösse von $R$ noch gar nicht weit von 1 verschieden, nämlich 0,848 (bei 0,1 Mf. 0,869 , bei 0,01 Mf. 0,944). Nach Nernst's Äusserungen über die Akkommodation ist absolut nicht einzusehen, warum dieselbe bei Kondensatorentladungen unvergleichlich stärker sein sollte als bei konstanten und Wechselströmen. Bis auf weiteres muss ich diese Umstände als erheblichen Einwand gegen die Nernstsche Theorie betrachten.

- Das Hauptergebnis dieser Theorie ist oben so ausgedrückt worden, dass bei jeder der in Rechnung gezogenen Reizarten das Zustandekommen der Erregung die Einwirkung einer bestimmten Energiesumme verlangt. Sollte sich dies wirklich definitiv bestätigen, so liegt doch gewiss die Auffassung sehr nahe, dass im Muskel die Zuckung durch eine bestimmte auf die Zeit verteilte Arbeit der auf der Nervenbahn anlangenden Erregung ausgelöst wird. Demgegenüber setzt die Nernst'sche Theorie stillschweigend voraus, dass zur Auslösung der Zuckung nichts weiter nötig ist als eine anlangende Nervenerregung von bestimmter Stärke, und dass diese Erregung an der Reizstelle gewissermassen fertig hergestellt wird in Gestalt der erreichten Konzentrationsgrösse; das oben S. 197 f. und 204 f. Ausgeführte 
218 L. Hermann: Untersuchungen über indirekte Muskelreizung etc.

zeigt zum mindesten, dass der letztere Standpunkt nicht etwa der einzig mögliche ist:

Übrigens erscheint eine experimentelle Entscheidung nicht ausgeschlossen. Bei einer Minimalzuckung durch einen, 0,003 Sekunde lang geschlossenen konstanten. Strom ist nach unseren bisherigen Vorstellungen der Moment der Schliessung der hauptsächlich erregende, nach der Nernst'schen Theorie dagegen würde die Reizung erst am Ende der Durchströmungsdauer erfolgen, weil erst dann die Konzentration die erforderliche Grösse erreicht hat; durch den Aktionsstrom wird sich vielleicht feststellen lassen, ob bei einem solchen Stromstoss der Abgang der Erregungswelle von der Reizstelle dem Moment der Schliessung oder dem der Öffnung näher liégt. 